\title{
Article
}

\section{Study of chemically peculiar stars - I. High-resolution spectroscopy and K2 photometry of Am stars in the region of M44}

Joshi, Santosh, Trust, Otto, Semenko, E, Williams, P E, Lampens, P, De Cat, P, Vermeylen, L, Holdsworth, Daniel Luke, García, R A, Mathur, S, Santos, A R G, Mkrtichian, D, Goswami, A, Cuntz, M, Yadav, A P, Sarkar, M, Bhatt, B C, Kahraman Aliçavus, F, Nhlapo, M D, Lund, M N, Goswami, P P, Savanov, I, Jorissen, A, Jurua, E, Avvakumova, E, Dmitrienko, E S, Chakradhari, N K, Das, M K, Chowdhury, S, Abedigamba, O P, Yakunin, I, Letarte, B and Karinkuzhi, D

Available at http://clok.uclan.ac.uk/40627/

Joshi, Santosh, Trust, Otto, Semenko, E, Williams, P E, Lampens, P, De Cat, P, Vermeylen, L, Holdsworth, Daniel Luke ORCID: 0000-0003-2002-896X, García, $R$ A et al (2022) Study of chemically peculiar stars - I. High-resolution spectroscopy and $K 2$ photometry of Am stars in the region of M44. Monthly Notices of the Royal Astronomical Society, 510 (4). pp. 5854-5871. ISSN 00358711

It is advisable to refer to the publisher's version if you intend to cite from the work. http://dx.doi.org/10.1093/mnras/stab3158

For more information about UCLan's research in this area go to http://www.uclan.ac.uk/researchgroups/ and search for <name of research Group>.

For information about Research generally at UCLan please go to http://www.uclan.ac.uk/research/

All outputs in CLoK are protected by Intellectual Property Rights law, including 


\section{CLoK}

Central Lancashire online Knowledge www.clok.uclan.ac.uk 


\title{
Study of chemically peculiar stars - I. High-resolution spectroscopy and $K 2$ photometry of Am stars in the region of M44
}

\author{
Santosh Joshi, ${ }^{1 \star}$ Otto Trust ${ }^{\oplus},{ }^{2 \star}$ E. Semenko, ${ }^{3,4}$ P. E. Williams, ${ }^{5}$ P. Lampens, ${ }^{6}$ P. De Cat ${ }^{\oplus}, 6$ \\ L. Vermeylen, ${ }^{6}$ D. L. Holdsworth ${ }^{\oplus},{ }^{7}$ R. A. García, ${ }^{8,9}$ S. Mathur, ${ }^{10,11}$ A. R. G. Santos, ${ }^{12,13}$ D. Mkrtichian, ${ }^{3}$ \\ A. Goswami, ${ }^{14}$ M. Cuntz, ${ }^{15}$ A. P. Yadav ${ }^{\circledR},{ }^{16}$ M. Sarkar, ${ }^{1}$ B. C. Bhatt, ${ }^{14}$ F. Kahraman Aliçavuş ${ }^{\circledR},{ }^{17,18}$ M. \\ D. Nhlapo, ${ }^{19}$ M. N. Lund ${ }^{\circledR},{ }^{20}$ P. P. Goswami, ${ }^{14}$ I. Savanov, ${ }^{21}$ A. Jorissen, ${ }^{22}$ E. Jurua, ${ }^{2}$ E. Avvakumova, ${ }^{23}$ \\ E. S. Dmitrienko, ${ }^{24}$ N. K. Chakradhari, ${ }^{25}$ M. K. Das,${ }^{26}$ S. Chowdhury, ${ }^{17}$ O. P. Abedigamba, ${ }^{19,27}$ \\ I. Yakunin, ${ }^{4}$ B. Letarte $^{19}$ and D. Karinkuzhi ${ }^{28}$
}

Affiliations are listed at the end of the paper

Accepted 2021 October 25. Received 2021 October 25; in original form 2021 April 16

\begin{abstract}
We present a study based on the high-resolution spectroscopy and $K 2$ space photometry of five chemically peculiar stars in the region of the open cluster M44. The analysis of the high-precision photometric $K 2$ data reveals that the light variations in HD 73045 and HD 76310 are rotational in nature and caused by spots or cloud-like co-rotating structures, which are non-stationary and short-lived. The time-resolved radial velocity measurements, in combination with the $K 2$ photometry, confirm that HD 73045 does not show any periodic variability on time-scales shorter than $1.3 \mathrm{~d}$, contrary to previous reports in the literature. In addition to these new rotational variables, we discovered a new heartbeat system, HD 73619, where no pulsational signatures are seen. The spectroscopic and spectropolarimetric analyses indicate that HD 73619 belongs to the peculiar Am class, with either a weak or no magnetic field, considering the 200-G detection limit of our study. The least-squares deconvolution profiles for HD 76310 indicate a complex structure in its spectra, suggesting that this star is either part of a binary system or surrounded by a cloud shell. When placed in the Hertzsprung-Russell diagram, all studied stars are evolved from the main sequence and situated in the $\delta$ Scuti instability strip. This work is relevant for further detailed studies of chemically peculiar stars, for example on inhomogeneities (including spots) in the absence of magnetic fields and the origin of the pulsational variability in heartbeat systems.
\end{abstract}

Key words: techniques: photometric-spectroscopic-polarimetric-stars: chemically peculiar-stars: activity -stars: binaries.

\section{INTRODUCTION}

The chemically peculiar (CP) stars are a group of main-sequence B-, A-, and F-type stars having peculiar surface elemental abundances; they are characterized by abnormal spectral line strengths (Preston 1974). The chemical anomalies in these stars are thought to be confined to the outer stellar layers and to arise from gravitational settling and radiative levitation of certain elements, a process known as atomic diffusion (Michaud 1970; Michaud, Charland \& Megessier 1981). This study is confined to one subset of CP stars, the metalliclined A (Am) stars, which are generally non-magnetic in nature and characterized by under-abundances of some light elements such as $\mathrm{Ca}$ and $\mathrm{Sc}$, but with slight/moderate over-abundances of iron-peak elements, for example $\mathrm{Zn}, \mathrm{Sr}, \mathrm{Y}, \mathrm{Zr}$, and $\mathrm{Ba}$. The projected rotational velocities of these stars are generally smaller than for ordinary A stars ( $v \sin i$ typically $<120 \mathrm{~km} \mathrm{~s}^{-1}$ ), with the majority of the Am stars being members of close binary systems. Rotational braking through tidal interaction is regarded as a possible cause of the low rotational velocities.

^E-mail: santosh@aries.res.in (SJ); otrust@ must.ac.ug (OT)
Using four years of high-precision photometry from the nominal Kepler mission and the $K 2$ campaigns, Balona et al. (2015) investigated the light variations in 29 Am stars and found that most of the Am stars in the Kepler field have light curves with the characteristics of rotational modulation arising from star spots or co-rotating structures. The origin of spots in Am stars seems to be different from that of solar-like spots, as these stars do not show any signs of the intense magnetic fields able to produce such magnetic features. Magnetic fields of the order of sub-Gauss strengths have been reported in some Am stars, for example Sirius A (Petit et al. 2011), Vega (Böhm et al. 2015), $\beta$ UMa, and $\theta$ Leo (Blazère et al. 2015), Alhena (Blazère, Neiner \& Petit 2016) and $\rho$ Pup (Neiner, Wade \& Sikora 2017). It is thought that for the majority of these stars, convective flows in the atmospheres may disrupt any spot-like features (Kupka 2003). Hence, the rotational modulation in some Am stars indicates either that a weak magnetic field may lead to surface inhomogeneities in the form of spots across the stellar surface or, alternatively, that there is some unknown mechanism(s) producing these spots. If a weak magnetic field is indeed present, then the basic processes operating in Am stars would need to be revisited because magnetic fields have been omitted from the diffusion models attempting to explain their unusual chemical abundances (Guzik 2021). 
Table 1. The specifications of the telescopes and back-end instruments used for the photometric and spectroscopic observations.

\begin{tabular}{|c|c|c|c|c|c|}
\hline Category & $\begin{array}{l}\text { Name of } \\
\text { telescope }\end{array}$ & $\begin{array}{l}\text { Diameter } \\
\quad(\mathrm{m})\end{array}$ & Location & $\begin{array}{c}\text { Detector/ } \\
\text { Spectrograph }\end{array}$ & $\begin{array}{l}\text { FoV/ } \\
\text { Resolution }\end{array}$ \\
\hline \multirow[t]{4}{*}{$\begin{array}{l}\text { Ground-based } \\
\text { photometry }\end{array}$} & DFOT & 1.30 & Devasthal (India) & $\begin{array}{l}\text { Andor's DZ436 } \\
\text { Andor's iXon EM + DU-897 }\end{array}$ & $\begin{aligned} 18^{\prime} & \times 18^{\prime} \\
4.7^{\prime} & \times 4.7^{\prime}\end{aligned}$ \\
\hline & ST & 1.04 & Nainital (India) & 2k Wright CCD & $13^{\prime} \times 13^{\prime}$ \\
\hline & MASTER-II-URAL & 0.40 & Ural Federal University (Russia) & U16M CCD & $2^{\circ} \times 4^{\circ}$ \\
\hline & PROMPT-8 & 0.60 & CTIO (Chile) & Apogee, F42 & $26.6^{\prime} \times 22.6^{\prime}$ \\
\hline \multirow[t]{4}{*}{ Spectroscopy } & BTA & 6.00 & Nizhniy Arkhyz (Russia) & NES,MSS & 39000,15000 \\
\hline & TNO & 2.40 & Chiang Mai (Thailand) & MRES & 17000 \\
\hline & $\mathrm{HCT}$ & 2.00 & Hanle (India) & HESP & 30000 \\
\hline & Mercator & 1.20 & La Palma (Spain) & HERMES & 85000 \\
\hline
\end{tabular}

Stars showing variable amplitudes or harmonics in their light curves provide the best cases for identifying rotational modulation. However, it is extremely difficult to distinguish between rotation and binarity at low frequencies, as the amplitude of the periodic variation due to co-rotating star spots changes on a relatively short time-scale (perhaps a few months).

Short-term variability, similar to that seen in $\delta$ Scuti stars, has been reported in many Am stars and is understood to arise from pulsations driven by the $\kappa$-mechanism operating in the He II ionization zone (Pamyatnykh 2000) combined with turbulent pressure in the $\mathrm{H} / \mathrm{He}$ I ionization zone (Smalley et al. 2017; Antoci et al. 2019). In the context of this study, Joshi et al. (2015) suspected a short-term pulsational variability in HD 73045, which is awaiting confirmation.

In close binary systems, stellar pulsations may also be tidally induced (Kumar, Ao \& Quataert 1995; Thompson et al. 2012), for example as in KOI-54 (Welsh et al. 2011; Fuller \& Lai 2012; Burkart et al. 2012; O'Leary \& Burkart 2014) and KIC 3230227 (Guo, Gies \& Fuller 2017). Highly eccentric $(e>0.3)$ binary systems with orbital periods between a fraction of a day and tens of days showing a sudden increase in brightness at periastron passage, of the order of several parts per thousand (ppt), are known as heartbeat (HB) stars (Handler et al. 2002; Maceroni et al. 2009; Hambleton et al. 2013; Beck et al. 2014; Hambleton et al. 2016, 2018; KołaczekSzymański et al. 2020). The name stems from the resemblance of the light curve to a heartbeat in an electrocardiogram. About $180 \mathrm{HB}$ stars have been discovered to date. Owing to their small-amplitude light variations and short orbital periods, these targets were mainly detected using space missions such as Kepler (Hambleton et al. 2013; Kirk et al. 2016; Hambleton et al. 2016; Fuller 2017; Hambleton et al. 2018; Guo et al. 2020). The Am stars are often part of binary systems (Carquillat \& Prieur 2007), although Am stars in HB systems have not yet been reported in the literature, and thus the discovery of a new HB system in one of the CP stars HD 73619 would be significant.

In this work, we revisit all stars previously observed as part of the Nainital-Cape survey project (Ashoka et al. 2000; Martinez et al. 2001; Joshi et al. 2003, 2006, 2009, 2010, 2012, 2016, 2017) with data in the Kepler and $K 2$ archives. It is our aim to search for low-frequency, high-precision, and photometric variability. No stars from the Nainital-Cape survey were observed in the nominal Kepler field, whilst eight stars were found in the $K 2$ archive. In this paper, we present the results on extrinsic (rotational and heartbeat) variables, as well as the results for stars where variability could not be ascertained.

\section{OBSERVATIONS AND DATA REDUCTION}

The photometric observations were obtained using both ground- and space-based telescopes, while spectroscopy and spectropolarimetry were performed through ground-based telescopes. The specifications of the telescopes and state-of-art instruments used for the observations are listed in Table 1.

\subsection{Ground-based differential photometry of HD 73045}

To investigate the variability of the single-lined spectroscopic binary (SB1) HD 73045, as suspected by Joshi et al. (2015), this object was extensively monitored photometrically with the 1.3-m Devasthal Fast Optical Telescope (DFOT; Sagar et al. 2011), the 1.04-m Sampurnanand Telescope (ST; Sinvhal et al. 1972), the $2 \times 0.40-$ $m$ MASTER-II-URAL telescope (Lipunov et al. 2010), and the 0.6-m Panchromatic Robotic Optical Monitoring and Polarimetry Telescope (PROMPT-8; Burdanov, Krushinsky \& Popov 2014). These instruments are detailed in Table 1.

All observations were carried out through a Johnson $B$ and/or $V$ filter. Calibration frames were acquired during each night for the correction of background and pixel sensitivity. The science frames were processed by subtracting master bias and dark frames before normalizing them with a median flat-field frame from which the cosmic rays were removed. The instrumental magnitudes of HD 73045 and the comparison stars (BD $+19^{\circ} 2046$ and TYC 1395-855-1) were obtained through aperture photometry using the in-built DAOPHOT package of IRAF (Davis 1994). The characteristics of the ground-based photometric observations are given in Table B1, and the individual light curves and power spectra are presented in Figs B1 and B2, in Appendix B. The top and bottom panels of Fig. 1 show the combined amplitude spectra of HD 73045 in the $B$ and $V$ filters, respectively.

\section{$2.2 \mathrm{~K} 2$ space photometry}

Our target stars were observed by the $K 2$ mission that operated in a long-cadence (LC; $29.4 \mathrm{~min}$ ) and a short-cadence (SC; $58.8 \mathrm{~s}$ ) mode (Howell et al. 2014). K2 pointed towards the Praesepe (Beehive) cluster (M44) during the campaigns C05 (2015/04/27-2015/07/10), C16 (2017/12/07-2018/02/25), and C18 (2018/05/12-2018/07/02). The $K 2$ observations of our targets are summarized in Table 2.

These photometric data sets were downloaded from the Barbara A. Mikulski Archive for Space Telescopes (MAST) data base ${ }^{1}$. Each

\footnotetext{
${ }^{1}$ https://archive.stsci.edu/k2/epic/search.php
} 


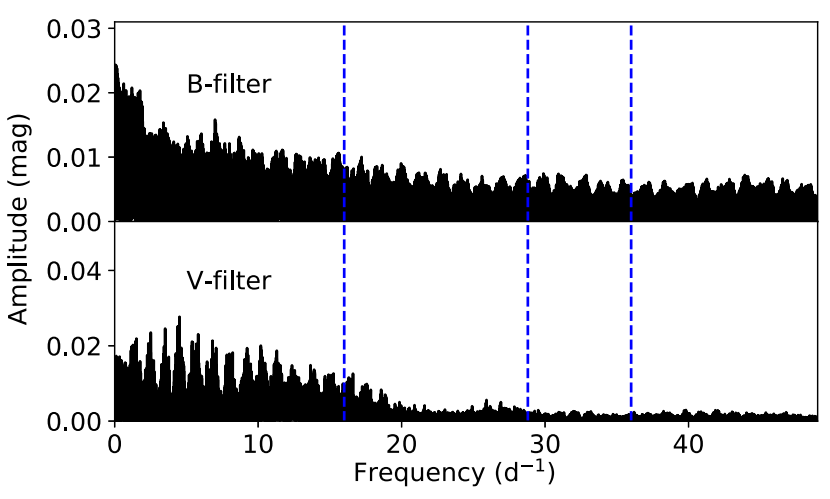

Figure 1. The amplitude spectra of HD 73045 using the combined data from ground-based observations in the $B$ (top panel) and $V$ (bottom panel) filters. There are no significant frequencies with signal-to-noise ratio (SNR) above 4 (0.048 mag for the $B$ filter and $0.051 \mathrm{mag}$ for the $V$ filter), and hence the star is classified as non-variable. The blue dashed vertical lines represent the short-term periodicity as suspected by Joshi et al. (2015).

Table 2. The $K 2$ campaigns concerning our programme stars.

\begin{tabular}{|c|c|c|c|c|c|c|c|}
\hline \multirow[t]{3}{*}{ Star name } & \multirow[t]{3}{*}{ EPIC number } & \multicolumn{6}{|c|}{$K 2$ observation campaign } \\
\hline & & \multicolumn{2}{|c|}{$\begin{array}{l}\mathrm{C} 05 \\
\sim 79 \mathrm{~d}\end{array}$} & \multicolumn{2}{|c|}{$\begin{array}{c}\mathrm{C} 16 \\
\sim 80 \mathrm{~d}\end{array}$} & \multicolumn{2}{|c|}{$\begin{array}{c}\text { C18 } \\
\sim 50 \mathrm{~d}\end{array}$} \\
\hline & & $\mathrm{LC}$ & $\mathrm{SC}$ & $\mathrm{LC}$ & SC & $\mathrm{LC}$ & SC \\
\hline HD 73045 & 211910450 & $\checkmark$ & - & - & - & $\checkmark$ & $\checkmark$ \\
\hline HD 73574 & 211989558 & $\checkmark$ & - & - & - & $\checkmark$ & - \\
\hline HD 73618 & 211955190 & $\checkmark$ & - & $\checkmark$ & - & $\checkmark$ & - \\
\hline HD 73619 & 211954496 & $\sqrt{ }$ & - & $\checkmark$ & - & $\checkmark$ & - \\
\hline HD 76310 & 212082764 & $\checkmark$ & - & $\checkmark$ & - & $\checkmark$ & - \\
\hline
\end{tabular}

target pixel file (TPF) was checked for the presence of additional stars. As HD 73045, HD 76310, and HD 73574 were the sole stars in their TPF images, their respective $K 2$ flexible image transport system (FITS) light-curve files were directly downloaded in the PDC_SAP format (Stumpe et al. 2012; Smith et al. 2012), having undergone presearch data conditioning (PDC) processing of their simple aperture photometry (SAP) flux data. The underlying position-dependent artefacts were removed by processing the data through the K2SC algorithm (Aigrain et al. 2015). The TPF images of HD 73618 and HD 73619 showed the presence of nearby stars (Fig. 2), which had to be masked out to isolate the flux of these target stars. The remaining images were averaged, and a custom mask was produced to isolate the target star. This mask was applied to each image of the campaign, and the total flux for each image was deduced, producing a light curve similar to the available SAP flux data. These data sets were further processed with the K2SC algorithm. Fig. 3 (top left-hand panel, in blue) shows an example light curve for the $\mathrm{C} 18$ observation of HD 73045. A detailed discussion of our analysis is given in Appendix A. A periodogram produced by the Lomb-Scargle algorithm is shown in Fig. 3 (bottom panel, in blue). The frequencyvarying artefact in the light curve is due to the roll-angle variation (Aigrain, Parviainen \& Pope 2016), reversing twice near 10 and 40 d, respectively. This artefact is not prevalent in the periodogram, but is distributed between 0.25 and $2 \mathrm{~d}\left(0.5-4 \mathrm{~d}^{-1}\right)$.

Using a multi-component sinusoidal model, frequencies, periods, amplitudes, and phases relating to the variability detected in the $K 2$ light curves were derived and are tabulated in Table 3. Fig. 3 (top left-hand panel, in red) illustrates such a model of the HD 73045 C18 light curve, for which the derived amplitudes and frequencies
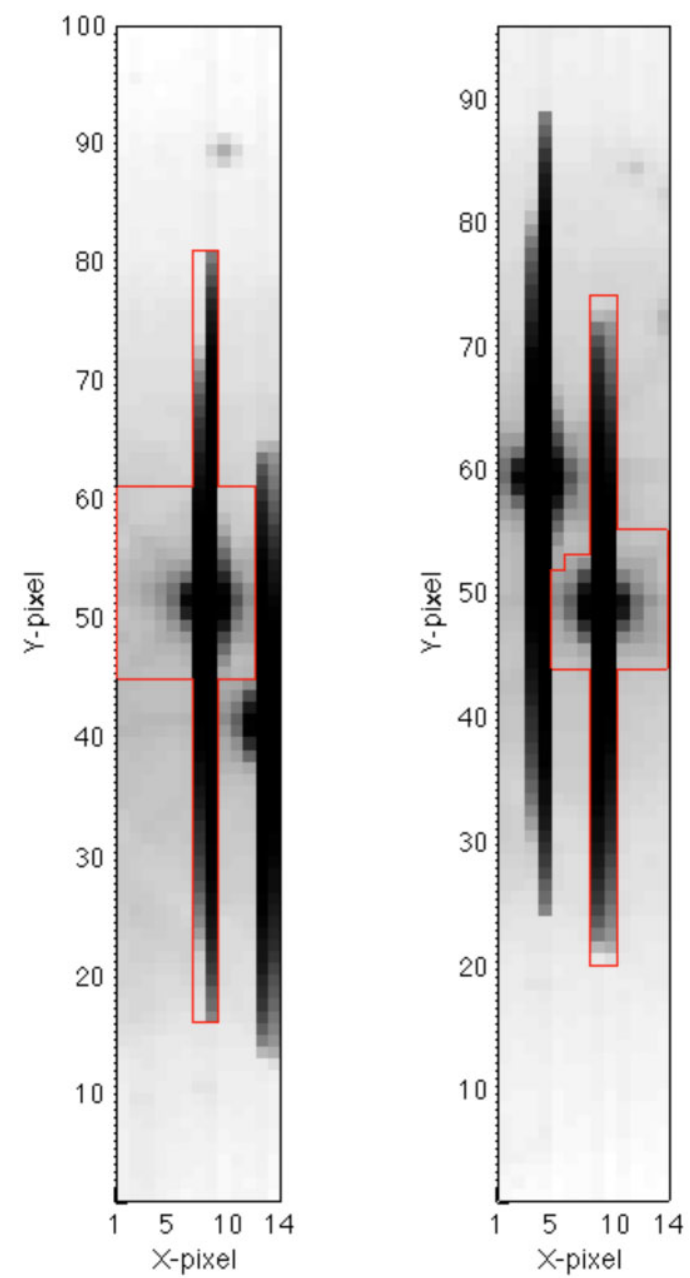

Figure 2. Target pixel images from the C18 time-series of HD 73618 (lefthand panel) and HD 73619 (right-hand panel). Note that each star appears in both images. The red border signifies the boundary of a custom mask within which the pixels are isolated and summed to produce a single simple aperture photometry flux data point.

of the components are included in the periodogram (Fig. 3, bottom panel) as red stems. The light-curve data are phase-folded on the period with the largest amplitude and overplotted with that period's sinusoidal component (Fig. 3, top right-hand panel). Similar figures for the remaining campaigns of all targets are given in Appendix B (Figs B7-B18).

\subsection{High-resolution spectroscopic and spectropolarimetric observations}

High-resolution spectroscopy provides an opportunity to derive a wide range of information on the target stars, for example basic stellar parameters such as the effective temperature $\left(T_{\text {eff }}\right)$, surface gravity $(\log g)$, and metallicity $([\mathrm{M} / \mathrm{H}])$. The high-resolution spectra of our targets were gathered with four spectrographs: (1) the Hanle Échelle Spectrograph (HESP; Chanumolu, Jones \& Thirupathi 2015; Sriram et al. 2018) at the 2.01-m Himalayan Chandra Telescope (HCT) of Indian Institute of Astrophysics (IIA), Bengaluru; (2) the Middle Resolution Échelle Spectrograph (MRES) at the 2.4m telescope of the Thai National Observatory (TNO) Thailand; (3) the High Efficiency and Resolution Mercator Échelle Spectrograph 

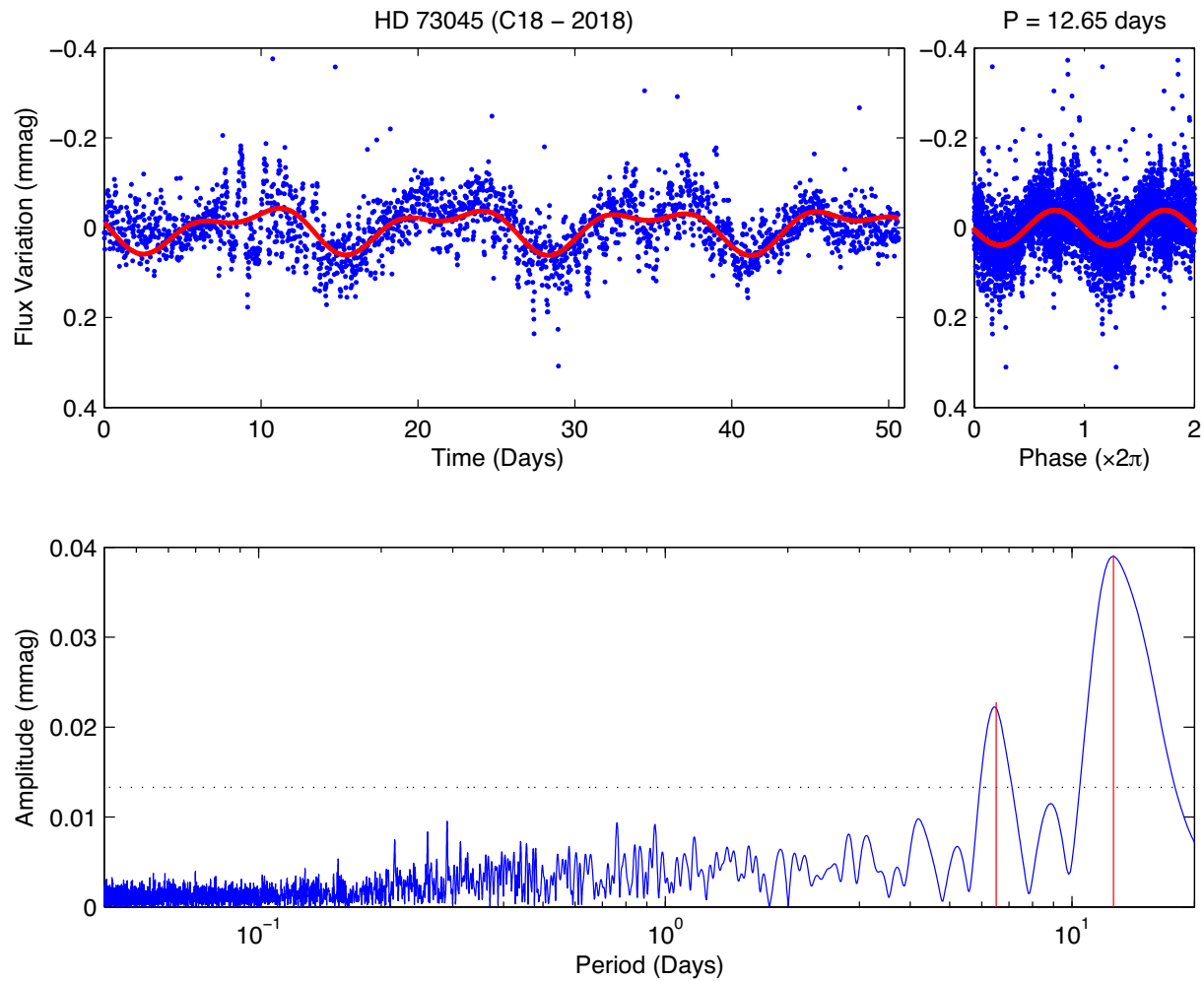

Figure 3. Top left-hand panel: Time-series illustrating photometric flux variation (in mmag) of HD 73045 for the LC 2018 K2 Campaign 18 . The data (blue points) include intrinsic signals as well as systemic and noise artefacts. The sinusoidal model, with parameters given in Table 3 , is shown as the red line. Top right-hand panel: The photometric data (blue points) phase-folded on the dominant variation period of $12.2 \mathrm{~d}$. The red line represents the best fit to these data. The data are repeated over two periods for clarity of presentation. Bottom panel: Linear-log periodogram of spectral amplitudes (blue line), in mmag. The components of the sinusoidal model are shown by the red stems. The black dotted line corresponds to a false alarm probability of $10^{-8}$, and the periodogram peaks above this line are considered to be confirmed signals.

(HERMES; Raskin et al. 2011) at the 1.2-m Mercator telescope; and (4) the Nasmyth Échelle Spectrometer (NES; Panchuk, Klochkova \& Yushkin 2017) at the 6-m Bolshoi Teleskop Alt-azimutalnyi (BTA) of the Special Astrophysical Observatory (SAO; Russia).

For HD 73619, we also used the Main Stellar Spectrograph (MSS; Chountonov 2004; Panchuk, Chuntonov \& Naidenov 2014) at the 6$\mathrm{m}$ BTA for measuring stellar magnetic field strengths. Our target was observed along with two standard stars, namely HD 52711 (non-magnetic) and $53 \mathrm{Cam}$ (magnetic). These spectropolarimetric observations were carried out in a series of paired exposures obtained in two orthogonal orientations of a retarder. The log of the highresolution spectroscopic and spectropolarimetric observations is summarized in Table 4.

Data reduction was performed using the pipeline developed for each particular spectrograph that generally involves overscan correction, averaged bias subtraction, flat-field correction, and wavelength calibration. The spectropolarimetric data reduction was performed using the ZEEMAN package developed within the ESO MIDAS environment for the specific format of CCD spectra taken with an image slicer (Kudryavtsev et al. 2006).

\section{RADIAL VELOCITY MEASUREMENTS}

We measured the radial velocities (RVs) of all target stars using the least-squares deconvolution (LSD) technique (Kochukhov, Makaganiuk \& Piskunov 2010). For HD 73045, the obtained RV time-series data were analysed using the standard discrete Fourier transform (DFT) technique. The results of the RV analysis for HD 73045 obtained from HERMES and MRES are shown in Figs B3 and B4, respectively. The frequency spectra at different epochs do not show any significant peak with $\mathrm{SNR}>4$. Therefore, we conclude that in the observational limit $\left(3 \sigma \sim 300 \mathrm{~m} \mathrm{~s}^{-1}\right)$, HD 73045 does not vary spectroscopically on a short time-scale. The RV of HD 73045 was also measured from the single spectrum from NES. All RV measurements are plotted with the orbital parameters given by Carquillat \& Prieur (2007), see Fig. B5. Based on the residuals shown in the bottom panel of Fig. B5, our results are consistent with the known orbital solution (with an orbital period of $435.57 \mathrm{~d}$ ).

For HD 73619, the RV measurements based on the MSS and HESP spectra (see Table 5) are fitted well using the orbital parameters (with an orbital period of $12.91 \mathrm{~d}$ ) derived by Debernardi et al. (2000). Fig. 4 shows the orbital solution of HD 73619 and compares the observations with the model. Our RV observations for both HD 73045 and HD 73619 agree with the known orbital parameters; hence, we did not re-derive them.

\section{ATMOSPHERIC PARAMETERS}

Much of the success of astronomy and astrophysics relies on accurate knowledge about the basic parameters and structure of stars. Using high-resolution spectroscopic data, HD 73045 has been extensively studied by Fossati et al. (2007, 2008); hence, we refrained from re-deriving its atmospheric parameters. To determine the relevant atmospheric parameters, for example $T_{\text {eff }}, \log g$, etc., for the remaining 
Table 3. Parameters of the sinusoidal component (ordered by amplitude), related to the variations observed within the analysed flux data, as derived from the fitting process. For each data set, these components have been summed to produce a model of their respective light curves.

\begin{tabular}{|c|c|c|c|c|c|}
\hline Star name & Campaign & $\begin{array}{l}\text { Frequency } \\
\qquad\left(d^{-1}\right)\end{array}$ & $\begin{array}{l}\text { Period } \\
\text { (d) }\end{array}$ & $\begin{array}{l}\text { Amplitude } \\
\text { (mmag) }\end{array}$ & $\begin{array}{l}\text { Phase } \\
\text { (rad) }\end{array}$ \\
\hline \multirow[t]{7}{*}{ HD 73045} & \multirow[t]{5}{*}{$\mathrm{C} 05$} & $0.0783 \pm 0.0002$ & $12.77 \pm 0.03$ & $0.113 \pm 0.003$ & $1.44 \pm 0.03$ \\
\hline & & $0.1555 \pm 0.0005$ & $6.43 \pm 0.02$ & $0.035 \pm 0.002$ & $-0.47 \pm 0.06$ \\
\hline & & $0.0640 \pm 0.0005$ & $15.6 \pm 0.1$ & $0.030 \pm 0.002$ & $-2.38 \pm 0.07$ \\
\hline & & $0.1395 \pm 0.0006$ & $7.17 \pm 0.03$ & $0.026 \pm 0.002$ & $-1.31 \pm 0.08$ \\
\hline & & $0.1668 \pm 0.0008$ & $6.00 \pm 0.03$ & $0.020 \pm 0.002$ & $-0.9 \pm 0.1$ \\
\hline & \multirow[t]{2}{*}{$\mathrm{C} 18$} & $0.0791 \pm 0.0005$ & $12.64 \pm 0.08$ & $0.039 \pm 0.002$ & $-0.008 \pm 0.05$ \\
\hline & & $0.1535 \pm 0.0008$ & $6.51 \pm 0.03$ & $0.023 \pm 0.002$ & $-0.54 \pm 0.07$ \\
\hline \multirow[t]{15}{*}{ HD 76310} & \multirow[t]{6}{*}{$\mathrm{C} 05$} & $0.2165 \pm 0.0002$ & $4.618 \pm 0.004$ & $0.50 \pm 0.01$ & $2.81 \pm 0.02$ \\
\hline & & $0.2039 \pm 0.0002$ & $4.905 \pm 0.005$ & $0.320 \pm 0.008$ & $-1.39 \pm 0.03$ \\
\hline & & $0.2290 \pm 0.0002$ & $4.367 \pm 0.005$ & $0.197 \pm 0.007$ & $0.19 \pm 0.03$ \\
\hline & & $0.1863 \pm 0.0003$ & $5.366 \pm 0.008$ & $0.149 \pm 0.006$ & $-0.84 \pm 0.04$ \\
\hline & & $0.4198 \pm 0.0004$ & $2.382 \pm 0.002$ & $0.106 \pm 0.005$ & $-2.56 \pm 0.05$ \\
\hline & & $0.1668 \pm 0.0004$ & $5.99 \pm 0.01$ & $0.099 \pm 0.004$ & $-2.75 \pm 0.05$ \\
\hline & \multirow[t]{6}{*}{$\mathrm{C} 16$} & $0.2089 \pm 0.0002$ & $4.786 \pm 0.004$ & $0.40 \pm 0.01$ & $-2.22 \pm 0.02$ \\
\hline & & $0.1856 \pm 0.0002$ & $5.388 \pm 0.005$ & $0.315 \pm 0.008$ & $-0.65 \pm 0.02$ \\
\hline & & $0.0756 \pm 0.0004$ & $13.23 \pm 0.07$ & $0.099 \pm 0.006$ & $0.90 \pm 0.06$ \\
\hline & & $0.4302 \pm 0.0004$ & $2.325 \pm 0.002$ & $0.084 \pm 0.005$ & $-2.51 \pm 0.06$ \\
\hline & & $0.9699 \pm 0.0004$ & $1.0310 \pm 0.0005$ & $0.080 \pm 0.005$ & $-2.25 \pm 0.06$ \\
\hline & & $0.0573 \pm 0.0005$ & $17.4 \pm 0.2$ & $0.065 \pm 0.005$ & $2.95 \pm 0.08$ \\
\hline & \multirow[t]{3}{*}{$\mathrm{C} 18$} & $0.2065 \pm 0.0002$ & $4.843 \pm 0.006$ & $1.09 \pm 0.02$ & $-1.05 \pm 0.02$ \\
\hline & & $0.1841 \pm 0.0005$ & $5.43 \pm 0.02$ & $0.25 \pm 0.01$ & $-2.47 \pm 0.04$ \\
\hline & & $0.4270 \pm 0.0006$ & $2.342 \pm 0.003$ & $0.18 \pm 0.01$ & $-2.4 \pm 0.06$ \\
\hline \multirow[t]{3}{*}{ HD 73574} & \multirow[t]{2}{*}{$\mathrm{C} 05$} & $0.0700 \pm 0.0004$ & $14.28 \pm 0.08$ & $0.85 \pm 0.04$ & $2.87 \pm 0.05$ \\
\hline & & $0.0925 \pm 0.0008$ & $10.8 \pm 0.1$ & $0.36 \pm 0.04$ & $1.7 \pm 0.1$ \\
\hline & $\mathrm{C} 18$ & $0.092 \pm 0.002$ & $10.8 \pm 0.2$ & $0.047 \pm 0.007$ & $0.4 \pm 0.1$ \\
\hline \multirow[t]{20}{*}{ HD 73618} & \multirow[t]{6}{*}{$\mathrm{C} 05$} & $0.2644 \pm 0.0002$ & $3.782 \pm 0.003$ & $0.40 \pm 0.01$ & $1.89 \pm 0.03$ \\
\hline & & $0.2055 \pm 0.0002$ & $4.867 \pm 0.006$ & $0.248 \pm 0.008$ & $0.90 \pm 0.03$ \\
\hline & & $0.4100 \pm 0.0004$ & $2.439 \pm 0.002$ & $0.136 \pm 0.007$ & $2.98 \pm 0.05$ \\
\hline & & $0.2531 \pm 0.0005$ & $3.951 \pm 0.008$ & $0.094 \pm 0.006$ & $2.78 \pm 0.07$ \\
\hline & & $0.4287 \pm 0.0005$ & $2.333 \pm 0.003$ & $0.092 \pm 0.006$ & $1.00 \pm 0.07$ \\
\hline & & $0.1766 \pm 0.0006$ & $5.66 \pm 0.02$ & $0.077 \pm 0.006$ & $-1.81 \pm 0.08$ \\
\hline & \multirow[t]{8}{*}{$\mathrm{C} 16$} & $0.2609 \pm 0.0002$ & $3.833 \pm 0.003$ & $0.32 \pm 0.009$ & $1.28 \pm 0.03$ \\
\hline & & $0.2052 \pm 0.0002$ & $4.874 \pm 0.004$ & $0.268 \pm 0.007$ & $2.74 \pm 0.03$ \\
\hline & & $0.4013 \pm 0.0003$ & $2.493 \pm 0.002$ & $0.151 \pm 0.005$ & $-2.03 \pm 0.04$ \\
\hline & & $0.2244 \pm 0.0003$ & $4.456 \pm 0.005$ & $0.125 \pm 0.005$ & $2.86 \pm 0.04$ \\
\hline & & $0.4167 \pm 0.0003$ & $2.399 \pm 0.002$ & $0.099 \pm 0.004$ & $2.51 \pm 0.05$ \\
\hline & & $0.2365 \pm 0.0003$ & $4.228 \pm 0.006$ & $0.085 \pm 0.004$ & $-2.90 \pm 0.05$ \\
\hline & & $0.2877 \pm 0.0004$ & $3.476 \pm 0.005$ & $0.066 \pm 0.004$ & $-0.23 \pm 0.06$ \\
\hline & & $0.4471 \pm 0.0005$ & $2.237 \pm 0.002$ & $0.062 \pm 0.004$ & $1.49 \pm 0.06$ \\
\hline & \multirow[t]{6}{*}{$\mathrm{C} 18$} & $0.2656 \pm 0.0003$ & $3.765 \pm 0.004$ & $0.60 \pm 0.01$ & $-1.54 \pm 0.02$ \\
\hline & & $0.2120 \pm 0.0004$ & $4.717 \pm 0.009$ & $0.224 \pm 0.008$ & $1.47 \pm 0.04$ \\
\hline & & $0.1951 \pm 0.0005$ & $5.13 \pm 0.01$ & $0.174 \pm 0.007$ & $-0.80 \pm 0.04$ \\
\hline & & $0.4180 \pm 0.0004$ & $2.392 \pm 0.002$ & $0.173 \pm 0.007$ & $2.89 \pm 0.04$ \\
\hline & & $0.2373 \pm 0.0005$ & $4.22 \pm 0.01$ & $0.113 \pm 0.006$ & $2.11 \pm 0.05$ \\
\hline & & $0.2941 \pm 0.0005$ & $3.401 \pm 0.006$ & $0.111 \pm 0.005$ & $-0.38 \pm 0.05$ \\
\hline \multirow[t]{14}{*}{ HD 73619} & \multirow[t]{7}{*}{$\mathrm{C} 05$} & $0.0771 \pm 0.0002$ & $12.97 \pm 0.03$ & $0.394 \pm 0.009$ & $-2.01 \pm 0.02$ \\
\hline & & $0.1548 \pm 0.0002$ & $6.46 \pm 0.01$ & $0.208 \pm 0.007$ & $-2.91 \pm 0.03$ \\
\hline & & $0.2326 \pm 0.0003$ & $4.300 \pm 0.005$ & $0.144 \pm 0.006$ & $2.52 \pm 0.04$ \\
\hline & & $0.3104 \pm 0.0005$ & $3.221 \pm 0.005$ & $0.085 \pm 0.005$ & $1.45 \pm 0.06$ \\
\hline & & $0.3882 \pm 0.0006$ & $2.576 \pm 0.004$ & $0.059 \pm 0.005$ & $0.77 \pm 0.09$ \\
\hline & & $0.465 \pm 0.001$ & $2.152 \pm 0.005$ & $0.033 \pm 0.005$ & $0.3 \pm 0.1$ \\
\hline & & $0.545 \pm 0.001$ & $1.834 \pm 0.005$ & $0.025 \pm 0.005$ & $-2.1 \pm 0.2$ \\
\hline & \multirow[t]{7}{*}{$\mathrm{C} 18$} & $0.0775 \pm 0.0003$ & $12.92 \pm 0.05$ & $0.344 \pm 0.01$ & $-1.27 \pm 0.03$ \\
\hline & & $0.1525 \pm 0.0004$ & $6.46 \pm 0.02$ & $0.179 \pm 0.007$ & $-0.9 \pm 0.04$ \\
\hline & & $0.2294 \pm 0.0006$ & $4.36 \pm 0.01$ & $0.113 \pm 0.006$ & $-0.81 \pm 0.05$ \\
\hline & & $0.3063 \pm 0.006$ & $3.265 \pm 0.008$ & $0.074 \pm 0.005$ & $-0.77 \pm 0.07$ \\
\hline & & $0.384 \pm 0.001$ & $2.607 \pm 0.007$ & $0.049 \pm 0.005$ & $-0.6 \pm 0.1$ \\
\hline & & $0.462 \pm 0.002$ & $2.16 \pm 0.01$ & $0.023 \pm 0.005$ & $-0.5 \pm 0.2$ \\
\hline & & $0.540 \pm 0.003$ & $1.85 \pm 0.01$ & $0.017 \pm 0.005$ & $-0.2 \pm 0.3$ \\
\hline
\end{tabular}


Table 4. Summary of the high-resolution spectroscopic observations. Spectropolarimetric observations are listed in the last row (MSS BTA).

\begin{tabular}{|c|c|c|c|c|}
\hline \multicolumn{5}{|c|}{ Spectrograph telescope } \\
\hline Star & $\begin{array}{c}\operatorname{HJD}(2450000+) \\
\text { (d) }\end{array}$ & $\begin{array}{c}\text { Total no. } \\
\text { spectra }\end{array}$ & $\begin{array}{c}\text { Spectral } \\
\text { range }(\mathrm{nm})\end{array}$ & SNR \\
\hline \multicolumn{5}{|c|}{ MRES TNT } \\
\hline \multirow[t]{3}{*}{ HD 73045} & $7007.391-7007.467$ & 38 & $406-879$ & $33-310$ \\
\hline & $7009.408-7009.470$ & 29 & & (@ $572 \mathrm{~nm}$ ) \\
\hline & $7011.365-7011.470$ & 31 & & \\
\hline HD 76310 & 8954.116 & 1 & & \\
\hline \multicolumn{5}{|c|}{ NES BTA } \\
\hline HD 73045 & 7350.462 & 1 & $409-686$ & $\begin{array}{c}300 \\
\text { (@ } 555 \mathrm{~nm} \text { ) }\end{array}$ \\
\hline \multicolumn{5}{|c|}{ HERME Mercator } \\
\hline HD 73045 & $7490.375-7490.496$ & 30 & $390-893$ & $40-45$ \\
\hline & $7491.377-7491.425$ & 10 & & (@650nm) \\
\hline & $7512.375-7512.460$ & 22 & & \\
\hline \multicolumn{5}{|c|}{ HESP HCT } \\
\hline HD 73574 & 8473.186 & 1 & $350-1000$ & 143 \\
\hline HD 73619 & 8473.223 & 1 & & 96 \\
\hline HD 76310 & 8473.261 & 1 & & 100 \\
\hline HD 73618 & 8555.074 & 1 & & $\begin{array}{c}220 \\
\text { (@ } 550 \mathrm{~nm} \text { ) }\end{array}$ \\
\hline \multicolumn{5}{|c|}{ MSS BTA } \\
\hline HD 73619 & $8578.300-8578.330$ & 3 & $444-498$ & $150-200$ \\
\hline & $8579.293-8579.314$ & 3 & & $\begin{array}{c}100-250 \\
(@ 455 \mathrm{~nm})\end{array}$ \\
\hline
\end{tabular}

targets, we used two approaches, namely photometric calibrations and high-resolution spectral data. We obtained the fundamental atmospheric parameters from photometry using the Geneva, $u v b y \beta$, and 2MASS photometric systems (Cutri et al. 2003), as well as via data obtained from the General Catalogue of Photometric Data ${ }^{2}$ (Mermilliod, Mermilliod \& Hauck 1997). The reddening parameters $E(B-V)$ and $E(B 2-V 1)$ were obtained using a 3D dust-map (Green 2018; Green et al. 2019) and ratios of total-to-selective absorption computed by Crawford \& Mandwewala (1976), respectively. In Table 6, we compile the known and derived parameters of the programme stars based on multicolour photometry.

The fundamental atmospheric parameters determined from photometry were used as initial input to facilitate further analyses. Spectroscopic parameters were derived by comparing observed spectra with synthetic ones computed for the adopted atmospheric parameters using the $\chi^{2}$-minimization method (see Trust et al. 2021 for more detail). They used the radiative transfer code MOOG (Sneden et al. 2012), together with the line-list version 5 of the Gaia-ESO Survey (GES) (wavelength range of 475-685 nm) (Heiter et al. 2015), the solar abundances by Asplund et al. (2009), and the ATLAS9 ${ }^{3}$ atmospheric models of Castelli \& Kurucz (2003) through the integrated software ISPEC (Blanco-Cuaresma et al. 2014; BlancoCuaresma 2019). The atmospheric parameters estimated from the best fits between the calculated and observed spectra are listed in Table 5. The synthetic spectra based on the values of the atmospheric parameters fit the observed spectra well, as shown in Fig. 5.

For HD 73619, the HESP and MSS spectra clearly show the presence of two components (see Fig. 6 and the LSD profiles of Fig. 7). We derived the atmospheric parameters from HESP and

\footnotetext{
${ }^{2}$ http://obswww.unige.ch/gcpd/indexform.html

${ }^{3}$ http://www.stsci.edu/hst/observatory/crds/castelli_kurucz_atlas.html
}

MSS spectra using the 2D version of the GIRFIT code developed by Frémat et al. (2006). We reconstructed the composite spectra by searching for the best pair of Doppler shifts and synthetic spectra, one for each component, with solar-like composition in the spectral regions 439-450, 450-470, 470-500, and 510-550 nm. Fig. 6 shows a portion of the HESP spectrum (black) and the fit (red) using a two-component model. The radial velocities as well as the derived atmospheric parameters are listed in Table 5.

\section{MAGNETIC FIELD MEASUREMENT OF HD 73619}

Magnetic field determinations allow us to distinguish between the two types of CP stars of interest, namely Ap and Am stars. Although spots are thought to occur in the presence of intense magnetic fields, Balona (2013) attributed the photometric variability observed in some A-type stars to the rotational modulation of stellar surfaces with inhomogeneities in cases where the magnetic field is either weak or absent. The magnetic field can be detected through the Zeeman effect, where the left and right circular polarization spectra are shifted with respect to each other, with the shift being proportional to the longitudinal magnetic field averaged over the stellar disc (Joshi et al. 2012, and references therein).

We used three techniques to measure the magnetic fields: centre of gravity, regression, and LSD. A mean value of 1.23 was adopted for the effective Landé factor, which is slightly higher than the corresponding parameter in the original paper by Bagnulo et al. (2002). An example of the intensity and circularly polarized LSD profiles of HD 73619 is depicted in Fig. 7. The magnetic field measurements are summarized in Table 7.

In addition to the target star HD 73619, during the same nights we also observed HD 52711 and 53 Cam as non-magnetic and magnetic standard stars, respectively. For each standard star, two spectra were obtained, one per night of observation. Within the observational uncertainties, the measured values of the magnetic field in $53 \mathrm{Cam}$ are consistent with the published results (e.g. Hill et al. 1998; Kochukhov et al. 2004; Martínez-Arnáiz et al. 2010). Unlike the magnetic standard stars, a solar-type star such as HD52711 should not manifest a magnetic field within the typical $B_{z}$ measurement uncertainties. A spurious signal of circular polarization in one spectrum of HD 52711, obtained during the second night, is attributable to instrumental effects.

This signal, in combination with an incomplete separation of lines in the spectrum of HD73619, is the apparent cause of the measured negative longitudinal magnetic field of the star. To check the reliability of this result, we constructed a mask of not-blended lines belonging to the different components and measured the signal only within the mask. Taking into account this finding, we conclude that neither component of the HD 73619 system possesses a magnetic field above $200 \mathrm{G}$. Nevertheless, in light of multiple observations of the extremely weak magnetic fields seen in some Am stars, we propose additional high-resolution spectropolarimetric observations prior to any confirmation of magnetic field strengths at the subhundred Gauss level.

\section{SURFACE ROTATION}

As a part of the Nainital-Cape survey, a total of 337 Ap and Am stars were monitored to search for photometric variability, and most of them turned out to be non-variables. The most plausible reason is because the light variations (if any) are of low amplitude, and 
Table 5. Basic parameters of the components of HD 73619 and HD 76310 obtained through high-resolution spectroscopy. The numbers 1 and 2 are assigned to the primary and secondary components, respectively. The spectral coverage was $430-680 \mathrm{~nm}$. We assumed equal light factors for both components of HD 73619, whereas for HD 76310 a light ratio $\left(l_{1}\right)$ of 0.887 was used. The stars HD 73045, HD 73574, and HD 73618 are assumed to be single stars, as either they are truly single, or the secondary star does not contribute significantly to the lines of the spectrum.

\begin{tabular}{|c|c|c|c|c|c|c|c|c|c|}
\hline \multirow[t]{2}{*}{ Star } & \multirow[t]{2}{*}{ Spectrograph } & \multicolumn{2}{|c|}{$\begin{array}{c}\mathrm{RV} \\
\left(\mathrm{km} \mathrm{s}^{-1}\right)\end{array}$} & \multicolumn{2}{|c|}{$\begin{array}{l}T_{\text {eff }} \\
(\mathrm{K})\end{array}$} & \multicolumn{2}{|c|}{$\begin{array}{l}\log g \\
(\operatorname{cgs})\end{array}$} & \multicolumn{2}{|c|}{$\begin{array}{c}v \sin i \\
\left(\mathrm{~km} \mathrm{~s}^{-1}\right)\end{array}$} \\
\hline & & 1 & 2 & 1 & 2 & 1 & 2 & 1 & 2 \\
\hline \multirow[t]{3}{*}{ HD 73619} & HESP & $-8.6 \pm 0.1$ & $77.5 \pm 0.1$ & $7150 \pm 90$ & $7220 \pm 100$ & $3.28 \pm 0.60$ & $3.02 \pm 0.12$ & $14.5 \pm 0.5$ & $13.3 \pm 0.8$ \\
\hline & MSS & $30.2 \pm 4.8$ & $46.8 \pm 3.3$ & $7410 \pm 370$ & $7200 \pm 280$ & $3.90 \pm 0.13$ & $3.54 \pm 0.22$ & $17.0 \pm 2.0$ & $19.5 \pm 0.5$ \\
\hline & & $12.3 \pm 1.5$ & $62.4 \pm 1.2$ & $7380 \pm 390$ & $7080 \pm 260$ & 4.0 & 4.0 & $16.3 \pm 4.0$ & $17.3 \pm 3.8$ \\
\hline \multirow[t]{2}{*}{ HD 76310} & HESP & $18.9 \pm 2.3$ & $10.1 \pm 1.8$ & $7420 \pm 210$ & $6220 \pm 180$ & 4.0 & 4.0 & $96 \pm 7$ & $9 \pm 1$ \\
\hline & MRES & $27.4 \pm 1.8$ & $19.8 \pm 2.8$ & $7030 \pm 170$ & $6470 \pm 220$ & 4.0 & 4.0 & $103 \pm 8$ & $20.3 \pm 3.4$ \\
\hline HD 73045* & ESPaDOnS & 27.9 & - & $7570 \pm 200$ & - & $4.05 \pm 0.2$ & - & $10 \pm 0.5$ & \\
\hline HD 73574 & HESP & $29.2 \pm 2.8$ & - & $7700 \pm 160$ & - & $4.12 \pm 0.21$ & - & $99 \pm 5$ & \\
\hline \multirow[t]{2}{*}{ HD 73618* } & ESPaDOnS & 15.1 & - & $8170 \pm 200$ & - & $4.00 \pm 0.19$ & - & $47 \pm 3$ & \\
\hline & HESP & $39.2 \pm 0.5$ & - & $7960 \pm 180$ & - & $3.76 \pm 0.19$ & - & $56 \pm 3$ & \\
\hline
\end{tabular}

Note. $*$ Fossati et al. (2007)

thus undetectable from the ground. Using the high-precision spacebased $K 2$ data, we confirmed the presence of submillimagnitude amplitude light variability in HD 73045, HD 73574, HD 73618, HD 73619, and HD76310, which were previously classified as non-variable stars as part of the Nainital-Cape survey. In the following subsections, we discuss the possible sources of light variation.

\subsection{Rotational modulation}

Rotational modulation in the light curve of a periodic variable is an indication of the presence of star spots or co-rotating clouds. The periodogram associated with rotational modulation can be characterized by the presence of harmonics of the rotation period (e.g. Santos et al. 2017). However, rotational variables may not always exhibit detectable harmonics in addition to the fundamental period. Therefore, at low frequencies, it becomes increasingly difficult to distinguish between binarity and rotation. Nevertheless, the amplitude of light variations due to co-rotating star spots may change in time because of spot evolution, whereas the amplitude of light variations due to orbital motion is expected to remain unchanged during the time of observation.

In order to understand the nature of low-frequency variations in A-type stars, Balona (2011) assumed that the frequency of highest amplitude in the frequency range $0.1 \mathrm{~d}^{-1}<f<5.0 \mathrm{~d}^{-1}$ is the rotation frequency. There are two ways of validating that the variability is due to rotational modulation. One way is to demonstrate that there is a relationship between the projected rotational velocities, $v \sin i$, and the predicted equatorial rotational velocities, $v_{\text {rot }}$. Because $\sin i \leq$ 1 , the expectation is that in a plot of $v \sin i$ as a function of $v_{\text {rot }}$, all points will lie below or (less frequently) on the line $v \sin i=v_{\text {rot }}$, subject to measurement errors (Balona 2017). Another method is to show that for stars in the main-sequence band the distribution of $v_{\text {rot }}$, derived from the rotational frequency $f_{\text {rot }}$ and from an estimate of the stellar radius, matches the distribution of $v_{\text {rot }}$ derived from spectroscopic measurements of $v \sin i$ for stars in the same stellar effective temperature range (Balona 2013).

Fig. 8 shows the distribution of $v \sin i$ as a function of $v_{\text {rot }}$. From this figure, it is evident that two stars, namely HD 73045 and HD 76310 , are located below the line corresponding to the inclination angle $i=90^{\circ}$. Therefore, the observed variations are compatible with an interpretation of spot-like features. Based on the two observations available to us, we classify HD 73045 and HD 76310 as rotational
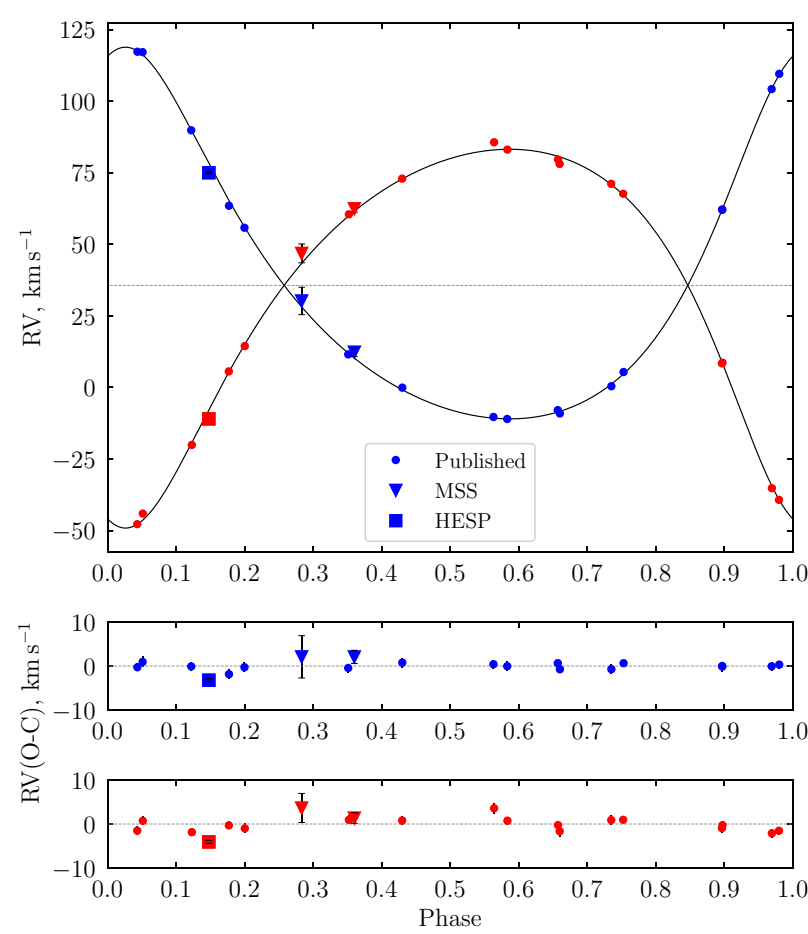

Figure 4. Radial velocity measurements of HD 73619 derived from both the HESP and MSS spectra are plotted together with the orbital solution given by Debernardi et al. (2000). Blue and red symbols represent the measured RVs of the primary and the secondary component, respectively.

variables. For the remaining two stars, HD 73574 and HD 73618, positioned above the diagonal line, the source of variability is unknown, and thus further investigation is required. We did not include HD 73619 in Fig. 8 because its dominant signal is clearly of orbital origin.

\subsection{Surface mapping}

In order to confirm the presence and locations of spots on stellar surfaces, the phased light curves were transformed into stellar images using the inversion technique developed by Savanov \& Strassmeier (2008). The surface mapping was performed for HD 73045 and 
Table 6. Basic parameters of the studied stars extracted from various online data bases. We list the atmospheric parameters $\left(T_{\text {eff }}, \log g\right.$, and $\left.[\mathrm{M} / \mathrm{H}]\right)$ computed from three photometric systems (Geneva, $u v b y \beta$ and 2MASS) as well as $\log \left(L_{\star} / \mathrm{L}_{\odot}\right)$ as calculated from the Gaia parallaxes.

\begin{tabular}{|c|c|c|c|c|c|c|c|c|c|c|c|c|c|}
\hline Star & $\begin{array}{c}V^{\mathrm{a}} \\
\text { (mag) }\end{array}$ & $\begin{array}{c}\pi^{\mathrm{b}} \\
\text { (mas) }\end{array}$ & $\begin{array}{c}E(B-V) \\
(\mathrm{mag})\end{array}$ & $\begin{array}{c}E(B 2-V 1) \\
(\mathrm{mag})\end{array}$ & $\begin{array}{c}T_{\text {eff }} \\
\text { Geneva } \\
( \pm 70 \mathrm{~K})\end{array}$ & $\begin{array}{l}{[\mathrm{M} / \mathrm{H}]} \\
\text { Geneva } \\
\pm 0.08\end{array}$ & $\begin{array}{c}T_{\mathrm{eff}} \\
u v b y \beta \\
( \pm 200 \mathrm{~K})\end{array}$ & $\begin{array}{c}\log g_{u v b y \beta} \\
( \pm 0.10 \mathrm{cgs})\end{array}$ & $\begin{array}{c}{[\mathrm{M} / \mathrm{H}]} \\
u v b y \beta \\
\pm 0.13\end{array}$ & $\begin{array}{c}T_{\mathrm{eff}} \\
\text { 2MASS } \\
( \pm 190 \mathrm{~K})\end{array}$ & $\log \left[L_{*} / \mathrm{L}_{\odot}\right]$ & Sp. type ${ }^{c}$ & Peculiarity \\
\hline HD 73574 & 7.75 & 5.7064 & 0.018 & 0.0138 & 7870 & 0.16 & 7660 & 3.91 & 0.15 & 7730 & $1.24 \pm 0.17$ & A3 & - \\
\hline HD 73618 & 7.30 & 6.9834 & 0.015 & 0.0118 & 7940 & 0.33 & 8090 & 3.90 & 0.39 & 7910 & $1.27 \pm 0.13$ & A0 & $\mathrm{Am}^{\mathrm{d}, \mathrm{e}}$ \\
\hline HD 73619 & 7.52 & 5.4033 & 0.015 & 0.0120 & 7670 & 0.52 & 7890 & 4.17 & 0.47 & 7860 & $1.20 \pm 0.06$ & A0 & $\mathrm{Am}^{\mathrm{f}}$ \\
\hline HD 76310 & 8.52 & 5.6101 & 0.015 & 0.0117 & 7310 & 0.14 & 7470 & 4.09 & 0.25 & 7320 & $0.94 \pm 0.11$ & A2 & $\mathrm{Am}^{\mathrm{g}}$ \\
\hline
\end{tabular}

${ }^{\mathrm{a}} \mathrm{H} \emptyset \mathrm{g}$ et al. (2000), ${ }^{\mathrm{b}}$ Gaia Collaboration (2018), ${ }^{\mathrm{c}}$ Cannon \& Pickering (1993), ${ }^{\mathrm{d}}$ Bertaud (1960), ${ }^{\mathrm{e}}$ Rebeirot (1966), ${ }^{\mathrm{f}}$ Bidelman (1956), and ${ }^{\mathrm{g}}$ Cowley \& Cowley (1965a).
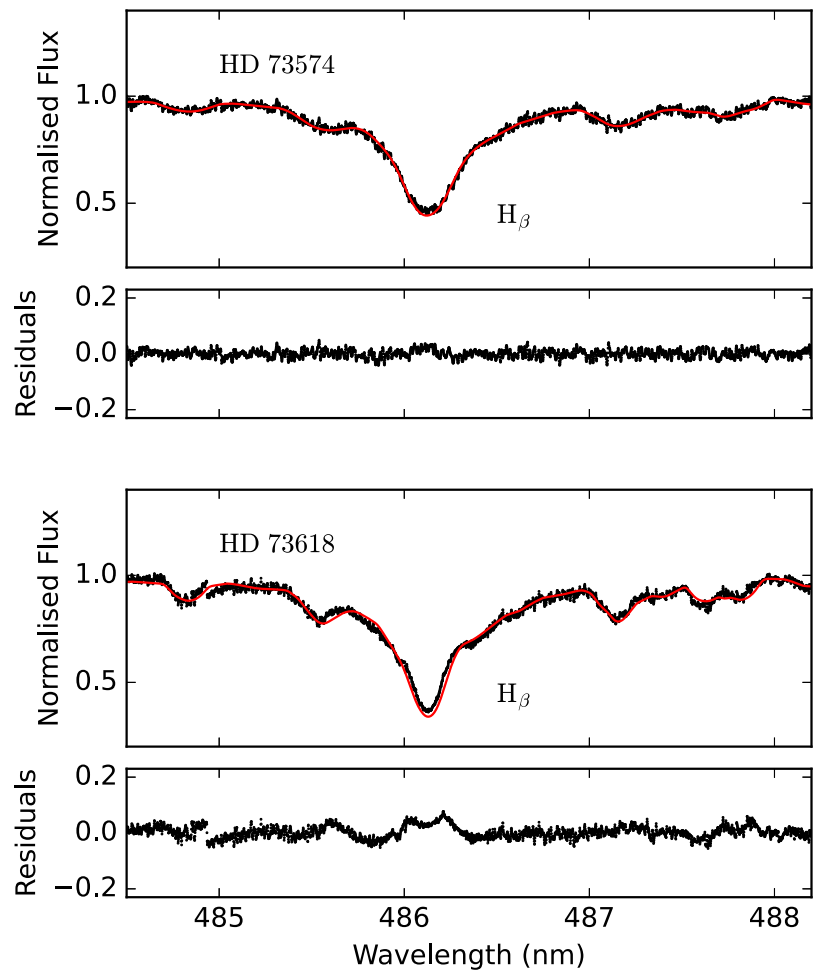

Figure 5. $\mathrm{H} \beta$ region of the spectra used for the determination of the respective stellar effective temperature. The synthetic spectrum (red) is overplotted on the observed spectrum (black).

HD 76310, classified as rotational variables in Section 6.1. The data for the other stars contain many artefacts that are most likely spacecraft-related (for example due to the regular thruster firings to maintain pointing), and hence the poor quality of the data prevents us from constructing high-precision spot maps.

The time-series data were divided into various observational data sets, each covering one rotational period of the star. Hence, we analysed 43 and 8 data sets for HD 76310 and HD 73045, respectively. The stellar surface was divided into a grid of $6^{\circ} \times 6^{\circ}$ pixels of unit area, and the values of the filling factor were determined for each grid pixel. Temperature-inhomogeneity maps were constructed for the inclination angle $i=45^{\circ}$.

Fig. 9 shows the surface-temperature-inhomogeneity maps of HD 73045. There are obvious concentrations of spots at two longitudes, registered as two independent spotted regions. The positions of the spots on the surface of HD 73045 vary rapidly on time-scales of one rotational period of about $13 \mathrm{~d}$. The maps clearly reveal that spots
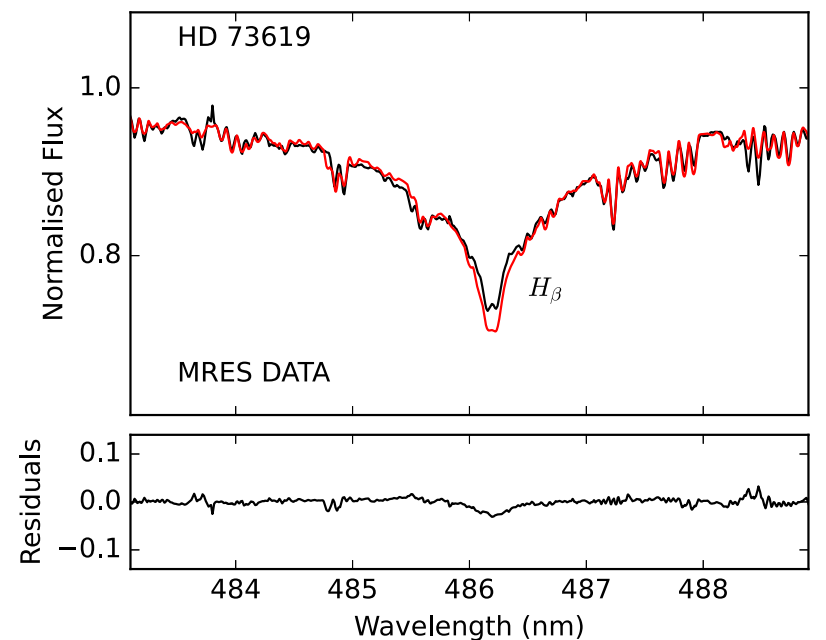

Figure 6. $\mathrm{H} \beta$ section of the MRES spectrum (black) for HD 73619 and the fit (red) using a two-component model and the $2 \mathrm{D}$ version of GIRFIT.

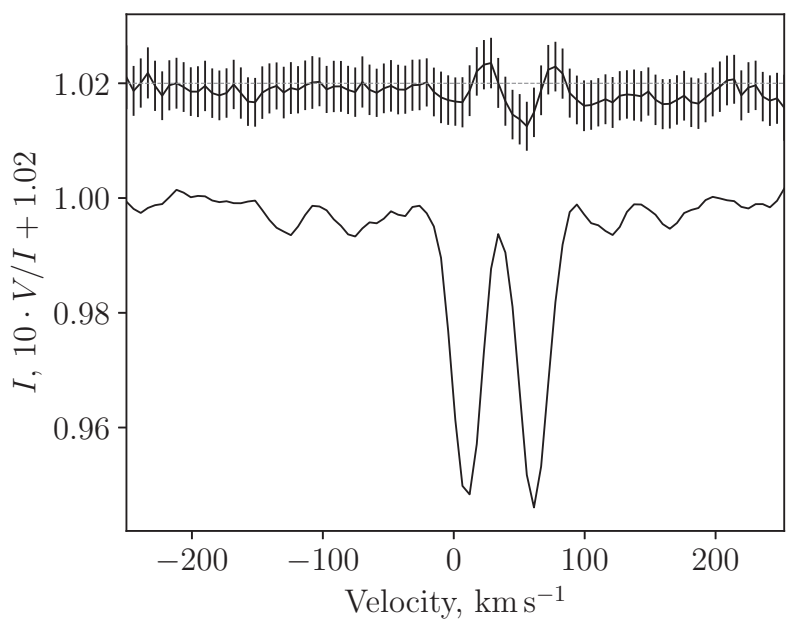

Figure 7. The intensity and circularly polarized LSD profiles of HD 73619.

are continuously changing their location in longitudinal direction, a clear indication of rotation. For HD 76310 (Fig. B6), there is typically only one spotted region at longitudes corresponding to phases $0.5-$ 0.7 , but for the map numbers 10 to $15,29,32$, and 35 , two spot-like structures are visible. Furthermore, the light curves become more flattened. The spot shape in most of the maps is elongated; one can also assume that for maps 10 to 15 , a single spotted region is split 
Table 7. Summary of measurements of the longitudinal magnetic field in HD 73619 and the standard stars. The third, fourth, and fifth columns represent the magnetic field measured using the classical centre of gravity, regression, and LSD profiles, respectively. The errors of the magnetic field are given by $\sigma$, and $n$ is the number of measured lines.

\begin{tabular}{lcccc}
\hline Star & $\begin{array}{c}\text { HJD }(2450000+) \\
(\mathrm{d})\end{array}$ & $\begin{array}{c}B_{z}^{\mathrm{g}} \pm \sigma(n) \\
(\mathrm{G})\end{array}$ & $\begin{array}{c}B_{z}^{\mathrm{r}} \pm \sigma \\
(\mathrm{G})\end{array}$ & $\begin{array}{c}B_{z}^{\mathrm{LSD}} \pm \sigma \\
(\mathrm{G})\end{array}$ \\
\hline HD 73619 & 8578.300 & $125 \pm 50(215)$ & $120 \pm 30$ & $70 \pm 50$ \\
& 8579.293 & $-150 \pm 50(82)$ & $-115 \pm 30$ & $-30 \pm 30$ \\
& & $-180 \pm 50(74)$ & $-110 \pm 25$ & \\
HD 52711 & 8578.321 & $3 \pm 50(267)$ & $10 \pm 20$ & $0 \pm 40$ \\
& 8579.307 & $170 \pm 50(244)$ & $150 \pm 40$ & $110 \pm 30$ \\
\multirow{2}{*}{53 Cam } & 8578.330 & $-4960 \pm 150(204)$ & $-3730 \pm 80$ & $-2837 \pm 50$ \\
& 8579.314 & $-3640 \pm 150(204)$ & $-2680 \pm 90$ & $-2170 \pm 50$ \\
\hline
\end{tabular}

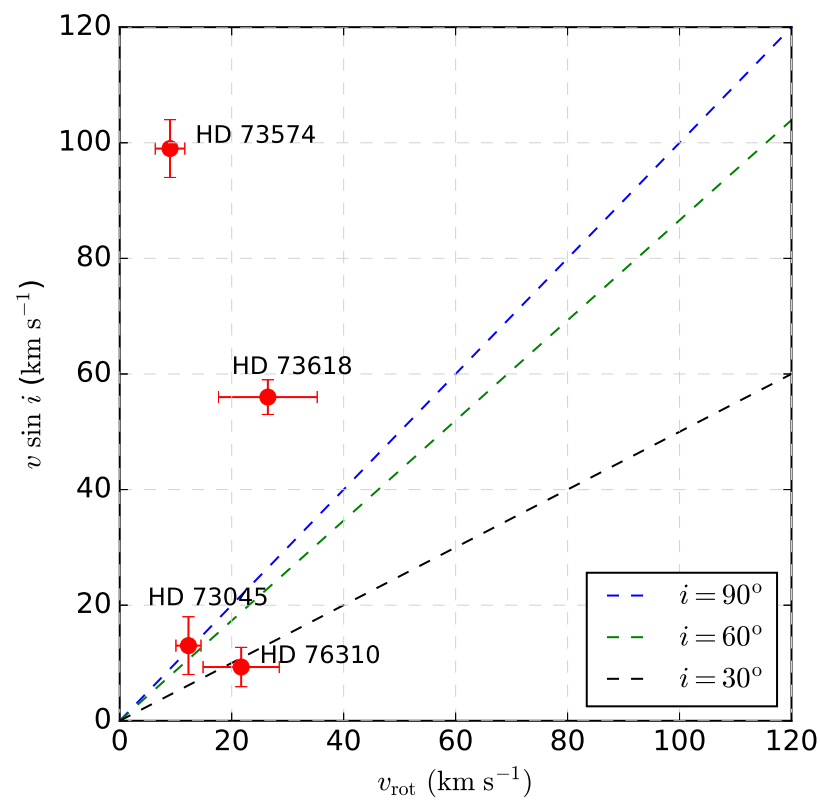

Figure 8. The projected rotational velocity $v \sin i$ as a function of predicted equatorial rotational velocity $v_{\text {rot }}$ for the target stars. The blue, green, and black dashed lines correspond to the inclination angles $i=90^{\circ}, 60^{\circ}$, and $30^{\circ}$, respectively. Note that HD 73619 is not included in this diagram because its photometric signal is orbital in nature.

into two regions. This result supports our previous detailed analysis of spot activity for several Am stars (Savanov 2018) exhibiting complex behaviours depending on the positions of spots on the stellar surfaces.

\subsection{Wavelet analysis}

Akin to sunspots, star spots are well-established tracers of stellar rotation, but their dynamic behaviour may also be used to analyse other relevant phenomena, such as stability, stellar magnetic activity, and cycles (García et al. 2010; Mathur et al. 2014). Similar to sunspots, the size and location of star spots also change; sometimes they suddenly appear or vanish. One might expect the same behaviour for spots on A-type stars, leading to variations in the amplitude of the frequencies. In order to investigate the change in frequency, we constructed time-frequency diagrams for the $K 2$ data sets of each star based on the wavelet technique, which allows a better interpretation of the physical features (such as spots) prior to their consideration for period refinement (Torrence \& Compo 1998; Mathur et al. 2010).
For a given signal, this technique permits the analysis of frequency (or period) variations with time (non-stationary signals). The Morlet wavelet, which is interpreted as the convolution of a sinusoidal and a Gaussian function, was used as the reference wavelet (Goupillaud, Grossmann \& Morlet 1984; Holschneider et al. 1989). For a given frequency, we calculated the correlation between the mother wavelet and the data by sliding the wavelet along the time axis of the light curves, resulting in a wavelet power spectrum (WPS). The WPS was then projected on the period axis to obtain the global wavelets power spectrum (GWPS). The time-frequency plots of the studied stars are shown in Fig. 10 and Figs B19-B28. The black and blue colours in the maps indicate regions of high and low power, respectively.

In all these cases, the variable (non-stationary) signal is noticeable. The variable signals in HD 73045 and HD 76310 could be due to the drifting of spot-like features or to spot growth and decay. For HD 73574, HD 73618 and HD 73619, variable signals are detected but do not seem to be of rotational origin (spots). To confirm the nature of the signal in HD 73574, a light curve with a longer time-scale is required, while that in HD 73618 seems to originate from the beating of close frequencies. The rotation periods $\left(P_{\mathrm{GWPS}}\right)$ extracted from the GWPS are $12.5 \pm 1.05$ and $4.8 \pm 0.45 \mathrm{~d}$ for HD 73045 and HD 76310, respectively. The detected periods $P_{\mathrm{GWPS}}$ for HD 73574, HD 73618, and HD 73619 are $14.0 \pm 1.05 \mathrm{~d}$, $3.8 \pm 0.44 \mathrm{~d}$, and $12.5 / 12.7 \pm 1.04 \mathrm{~d}$, respectively. The errors in the periods are derived from the errors in the frequencies, which are equivalent to the half-width at half-maximum (HWHM) of the peaks in the GWPS.

\subsection{Autocorrelation function analysis}

The surface mapping and the time-frequency analysis imprint the existence and evolution of spots on the surface of rotational variables. We autocorrelated the time-series data at a given time lag to reveal the spots possibly co-rotating with the stars (McQuillan, Aigrain \& Mazeh 2013; McQuillan, Mazeh \& Aigrain 2014; García et al. 2014; Santos et al. 2019). The positive and normalized resultant autocorrelation functions ( $\mathrm{ACF}$ ) have local maxima and minima that show the presence of spots on these stars; they mimic the displacement equation for an under-damped simple harmonic oscillator (uSHO) (Giles, Collier Cameron \& Haywood 2017). In each ACF, the first local maximum represents the dominant period $\left(P_{\mathrm{ACF}}\right)$. The $P_{\mathrm{ACF}}$ values for HD 73045, HD 73574, HD 73618, and HD 76310 are 12.8, 13.5, 4.1 , and 4.7 d, respectively, for the $K 2 \mathrm{C} 05$ data. These values are in good agreement with the periods derived from the amplitude spectra, wavelet maps, and composite spectra (e.g. Fig. 10 and Table 8).

The values of the spot variation time-scale $\left(\tau_{\mathrm{VT}}\right.$; related to the time of visibility of the spot on the stellar disc) obtained from fitting the ACF (C05) with an uSHO model are $18.67 \pm 0.38 \mathrm{~d}$ for HD 73045 and $19.31 \pm 0.13 \mathrm{~d}$ for HD 76310 . We note, however, that the dominant frequencies in the target stars, HD 73045 and HD 76310, are sustained for a duration longer than the $\tau_{\mathrm{VT}}$ as estimated from the ACF analysis. Santos et al. (2021) demonstrated that the $e$-folding time of the uSHO systematically underestimates the characteristic spot time-scale. Moreover, the length of the observations drastically limits the retrieved time-scale (Santos et al. 2021). As a result, the length of the $K 2$ time-series might be insufficient to constrain spot lifetimes through the ACF. Nevertheless, we list the values for $\tau_{\mathrm{VT}}$, which should be considered as lower limits, and we advise caution when interpreting the retrieved $\tau_{\mathrm{VT}}$. The additional values of $\tau_{\mathrm{VT}}$ obtained using the remainder of the campaigns are listed in Table 8.

Assuming that the amplitude of the rotational frequency is produced by a black circular spot, the scenario is identical to the 
0

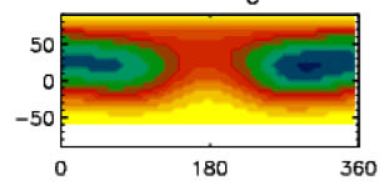

2

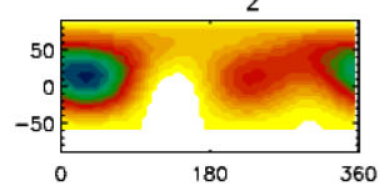

4

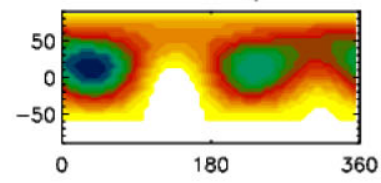

8

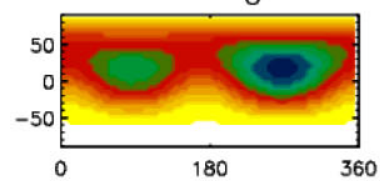

0

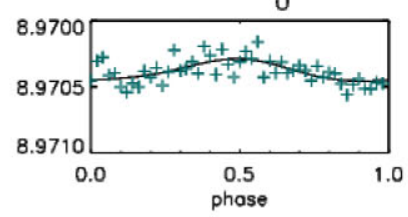

2

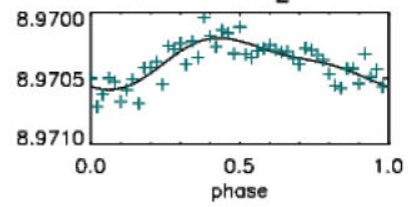

4

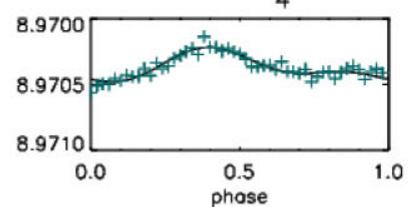

8

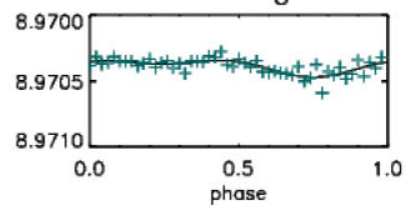

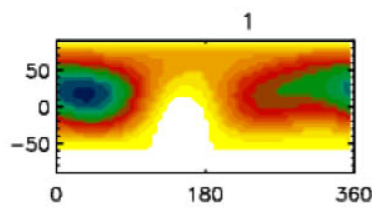

3

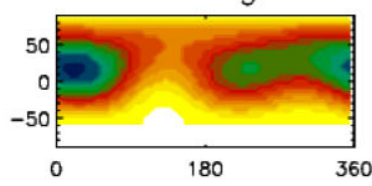

7

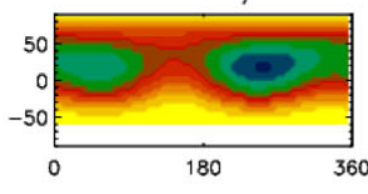

9

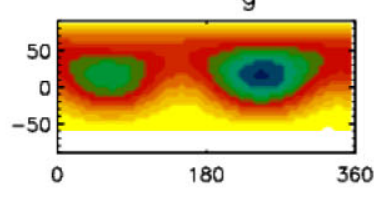

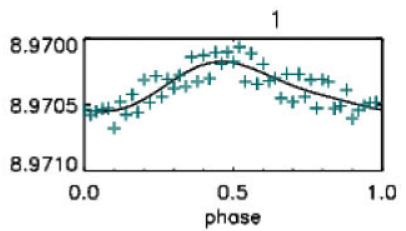

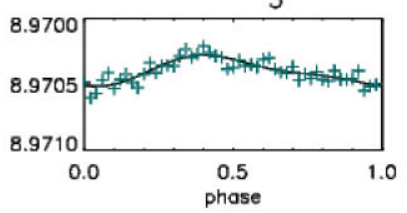

7
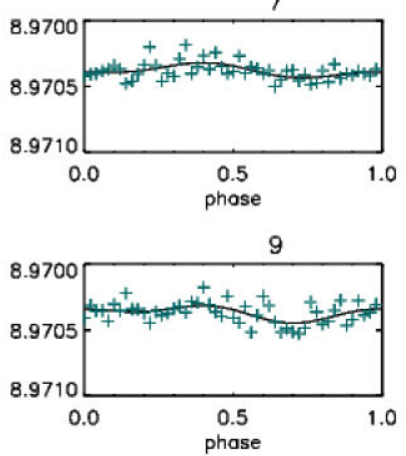

Figure 9. Temperature-inhomogeneity maps of HD 73045 constructed for eight data sets. All maps use the same scale. Darker areas imply a higher spot filling factor (i.e. the darkest regions correspond to a filling factor of about 0.994). The abscissa denotes the longitude in degrees, and the ordinate denotes the latitude in degrees. The phase diagrams show both the observed and the model reconstructed light curves.

determination of exoplanet sizes from a transit. Using K2 C05 data for HD 73045 and HD 76310, respectively, the minimum spot radius $\left(R_{\text {spot }}\right)$ - as derived from the photometric amplitude following a procedure described by Trust et al. (2020) - is $3.44 \pm 0.18 R_{\mathrm{E}}$ and $5.04 \pm 0.27 R_{\mathrm{E}}$, respectively (where $R_{\mathrm{E}}$ denotes the Earth's radius). The rest of the $R_{\text {spot }}$ values obtained through other campaigns, when available, are given in Table 8 . The $\tau_{\mathrm{VT}}$ of the spotted regions in these stars is of the order of a few weeks. Although, as discussed above, $\tau_{\mathrm{VT}}$ might not be a good constraint for the spot lifetimes owing to the length of the observations, these results might point towards weak or non-existent magnetic fields in the programme stars. The origin and nature of spots on such stars is a topic that will require detailed future modelling in consideration of this; for example, the star HD 73045 is known to be non-magnetic (Fossati et al. 2007) but shows unequivocal evidence of spots.

The composite spectrum (CS) combines the time-frequency analysis and the ACF, while being the product of the normalized GWPS and the normalized ACF (Ceillier et al. 2016, 2017). The period estimate, $P_{\mathrm{CS}}$, including the respective uncertainty, corresponds to the central period and HWHM of the Gaussian function that fits the highest peak in the CS. The results from the wavelet maps, and the ACF and CS analyses are listed in Table 8.

\section{AN OBSERVATIONAL \\ HERTZSPRUNG-RUSSELL DIAGRAM}

In order to infer the evolutionary status, the location of the target stars within the Hertszprung-Russell (H-R) diagram needs to be precisely determined. Therefore, accurate values for the effective temperature $T_{\text {eff }}$ and the luminosity $L_{\star}$ need to be obtained; note that a typical error of $150 \mathrm{~K}$ in $T_{\text {eff }}$ (as given by many traditional methods) entails an error of about 0.2 mag in the bolometric magnitude (Neiner \& Lampens 2015). Fortunately, high-resolution spectroscopy allows the deduction of accurate values of $T_{\text {eff }}$ and $\log \left(L_{\star} / \mathrm{L}_{\odot}\right)$, as this method takes into account the line blanketing caused by the chemical peculiarities.

The values of $\log \left(L_{\star} / \mathrm{L}_{\odot}\right)$ of the programme stars have been calculated using the standard relation as discussed in Balona (2018). The bolometric corrections for the Sloan Digital Sky Survey (SDSS) ugriz photometric system were obtained from Girardi et al. (2004). For the derivation of the absolute magnitude, the Gaia EDR3 parallaxes (Gaia Collaboration et al. 2018) were used. Combining all these values yielded adequate $\log \left(L_{\star} / L_{\odot}\right)$ values, which are presented in Table 6.

Based on the derived $T_{\text {eff }}$ and $\log \left(L_{\star} / \mathrm{L}_{\odot}\right)$ values, we placed the programme stars in the H-R diagram, and their locations are shown in Fig. 11. On inspection of their position, we conclude that all stars moved away from the zero-age main sequence (ZAMS) and are heading towards the terminal-age main sequence (TAMS). Considering the error boxes, all targets lie within the $\delta$ Scuti observational instability strip as determined by Murphy et al. (2019).

\section{COMMENTS ON INDIVIDUAL STARS}

Capitalizing on the unprecedented quality of photometric data obtained from space missions, searches for photometric variability followed by determinations of the various stellar physical properties using high-resolution spectroscopy help us to ascertain various stellar properties and activities such as chemical peculiarities, spots, pulsation, and rotation. The following subsections provide brief summaries and discussions about individual stars along with information on their basic physical properties. 

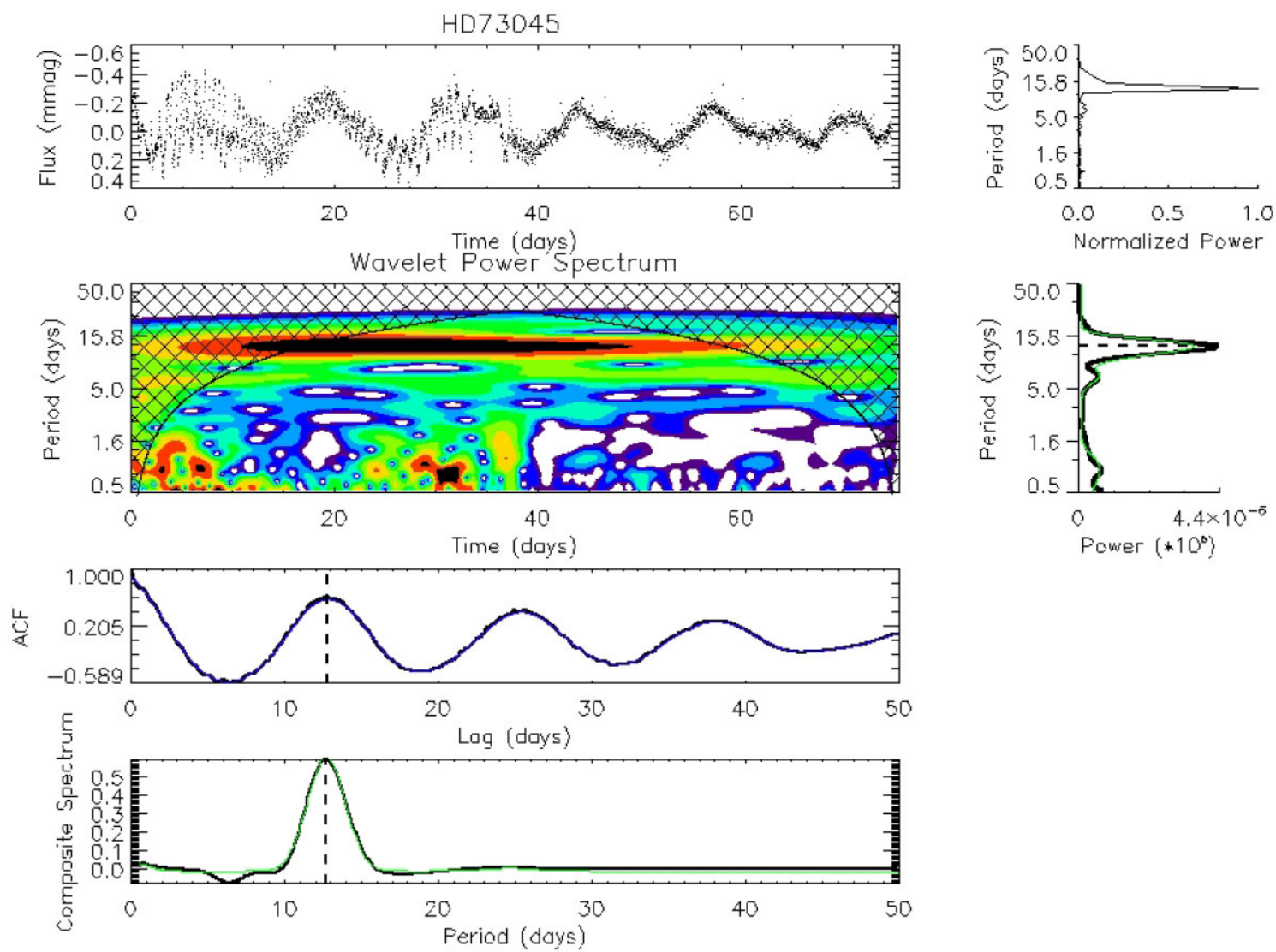

Figure 10. Wavelet map of HD 73045 using the C05 data. The top left-hand panel shows the light curve, while the top right-hand panel is the associated power density spectrum as a function of period between 0.5 and $50 \mathrm{~d}$. The left-hand panel of the second row depicts the wavelet power spectrum computed using a Morlet wavelet between 0.5 and $50 \mathrm{~d}$ on a logarithmic scale, and the associated global wavelet power spectrum is in the right-hand panel of the second row. The black and blue colours correspond to high and low power, respectively. The black-crossed area is the cone of influence corresponding to the unreliable results. The panel in the third row shows the autocorrelation function of the full light curve plotted between 0 and $50 \mathrm{~d}$. Finally, the composite spectrum (Ceillier et al. 2016, 2017) is shown in the bottom panel. The black dashed lines mark the respective rotation period estimates.

Table 8. The approximate spot radius $\left(R_{\text {spot }}\right)$ as derived from the amplitude, period $\left(P_{\mathrm{ACF}}\right)$, and variation time-scale $\left(\tau_{\mathrm{VT}}\right)$ as estimated from the autocorrelation functions and the rotation periods $\left(P_{\mathrm{GWPS}}\right)$. The composite spectrum power estimate $\left(P_{\mathrm{CS}}\right)$ is obtained from the respective global wavelet power spectrum and composite spectrum.

\begin{tabular}{|c|c|c|c|c|c|c|}
\hline Star & Campaign & $\begin{array}{l}R_{\text {spot }} \\
\left(R_{\mathrm{E}}\right)\end{array}$ & $\begin{array}{l}P_{\mathrm{ACF}} \\
\text { (d) }\end{array}$ & $\begin{array}{l}\tau_{\mathrm{VT}} \\
(\mathrm{d})\end{array}$ & $\begin{array}{l}P_{\mathrm{GWPS}} \\
(\mathrm{d})\end{array}$ & $\begin{array}{l}P_{\mathrm{CS}} \\
(\mathrm{d})\end{array}$ \\
\hline \multirow[t]{2}{*}{ HD 73045} & $\mathrm{C} 05$ & $3.44 \pm 0.18$ & 12.8 & $18.67 \pm 0.38$ & $12.5 \pm 1.04$ & $12.7 \pm 0.61$ \\
\hline & $\mathrm{C} 18$ & $2.02 \pm 0.17$ & 12.8 & $16.07 \pm 0.24$ & $12.5 \pm 1.05$ & $12.8 \pm 0.57$ \\
\hline HD 73574 & $\mathrm{C} 05$ & & 13.5 & & $14.0 \pm 1.05$ & $13.8 \pm 0.75$ \\
\hline \multirow[t]{3}{*}{ HD 73618} & $\mathrm{C} 05$ & & 4.1 & & $3.8 \pm 0.44$ & $3.9 \pm 0.21$ \\
\hline & $\mathrm{C} 16$ & & 4.3 & & $4.1 \pm 0.44$ & $4.3 \pm 0.23$ \\
\hline & $\mathrm{C} 18$ & & 3.9 & & $3.8 \pm 0.44$ & $3.8 \pm 0.20$ \\
\hline \multirow[t]{2}{*}{ HD 73619} & $\mathrm{C} 05$ & & 12.9 & & $12.7 \pm 1.04$ & $12.8 \pm 0.50$ \\
\hline & $\mathrm{C} 18$ & & 12.8 & & $12.5 \pm 1.04$ & $12.7 \pm 0.57$ \\
\hline \multirow[t]{3}{*}{ HD 76310} & $\mathrm{C} 05$ & $5.04 \pm 0.27$ & 4.7 & $19.31 \pm 0.13$ & $4.6 \pm 0.45$ & $4.7 \pm 0.21$ \\
\hline & $\mathrm{C} 16$ & $4.49 \pm 0.26$ & 4.9 & $18.55 \pm 0.27$ & $4.8 \pm 0.45$ & $4.9 \pm 0.23$ \\
\hline & $\mathrm{C} 18$ & $7.44 \pm 0.30$ & 4.8 & $21.68 \pm 0.13$ & $4.8 \pm 0.45$ & $4.8 \pm 0.22$ \\
\hline
\end{tabular}

\subsection{HD 73045/KW 538 (Praesepe)}

HD 73045 is classified as an Am star by Bidelman (1956) and Bertaud (1960) with a spectral type of A7.2 (Kraus \& Hillenbrand 2007) and is classified as an SB1 (Debernardi et al. 2000; Carquillat \& Prieur 2007). This star was extensively studied by Fossati et al. (2007, 2008), who derived the basic physical parameters and chem- ical composition. They reported that the star is non-magnetic in nature.

HD 73045 was photometrically analysed using the HI-1A photometer of the Heliospheric Imager (Eyles et al. 2009) onboard the STEREO spacecraft (Driesman, Hynes \& Cancro 2008) - a study done by Paunzen et al. (2013), who reported a variability period of $1.25 \mathrm{~d}$. However, this variation was a consequence of the pixel 


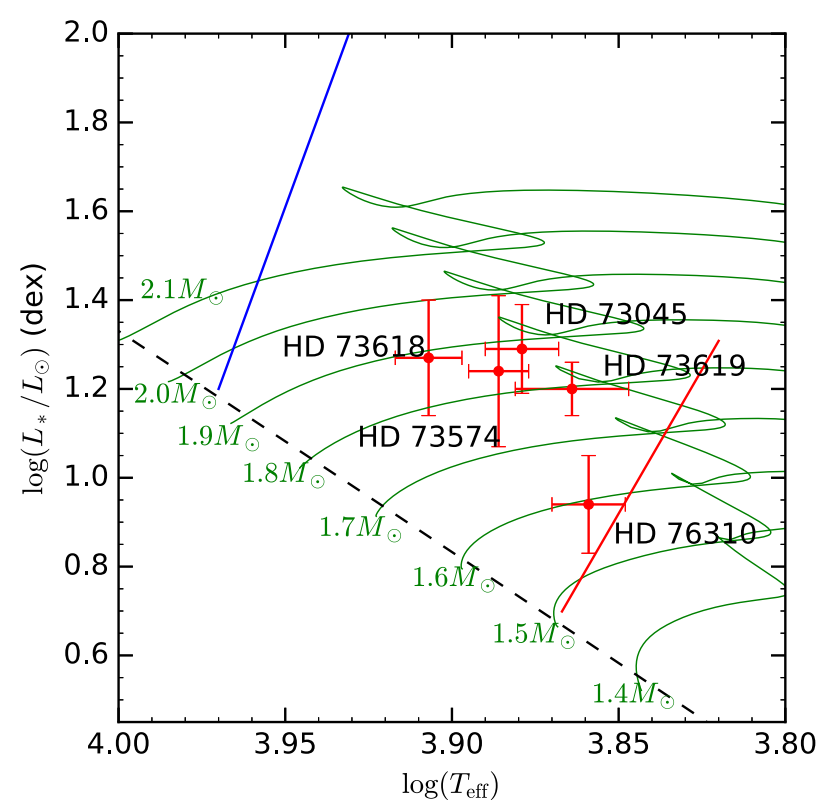

Figure 11. Location of the target stars in the H-R diagram. The solid green lines represent the theoretical evolutionary tracks for masses from $1.4 \mathrm{M}_{\odot}$ to $2.1 \mathrm{M}_{\odot}$ taken from Murphy et al. (2019). The blue and red edges of the observational $\delta$ Scuti instability strip are over-plotted with blue and red lines, respectively. The black dashed line represents the ZAMS.

response function (PRF) of HD 73045 blending with that of its visual neighbour $\mathrm{BD}+19^{\circ} 2046$ (Williams, private communication). The blended PRFs vary in shape and periodically bleed outside of the circular collection aperture as the sources track across the instrument's field-of-view. The sources were found to shift from one $y$-pixel to the next with a period of $1.25 \mathrm{~d}$, coinciding with the period reported by Paunzen et al. (2013). Our analysis did not find any evidence for a periodicity of $1.25 \mathrm{~d}$ either in the LC or in the SC $K 2$ data, hence contradicting the findings of the variability of $1.25 \mathrm{~d}$ reported by Paunzen et al. (2013).

Applying the analyses described in Section 2.2 to the LC $K 2$ data, the photometric variation exhibits a dominant component close to $12.65 \mathrm{~d}$ (12.68 d for C05 and $12.64 \mathrm{~d}$ for C18). The two data sets also show similar secondary signals at 6.42 and $6.51 \mathrm{~d}$, respectively. The WPS and GWPS (Fig. 10) also show an excess of power at around half of the dominant period. These secondary signals are close to harmonics of the dominant components. However, they do not correspond exactly to harmonics, as can be seen in the irregular characteristic of the light curve, highlighted by the models (e.g. Fig. 3 , top left-hand panel, red), whereas a harmonic signal would exhibit a repetitive period-to-period structure. This observed feature is also visible in other stars such as HD 174356 (Bowman et al. 2018) and, with more complexity, in Atlas in the Pleiades (White et al. 2017). Based on the surface map, time-frequency and ACF analyses, the origin of the periodic variability in HD 73045 could be attributable to star spots situated at different latitudes on a star exhibiting differential rotation. In particular, the rotational modulation in Fig. 3 shows a double-dip feature, which indicates the presence of at least two dominant spots.

On analysing the C18 SC data (Fig. B8, top), the two main components described above are observed. The main goal in studying these data, however, was to confirm the short-term variability (i.e. $16,28.8$, and $36 \mathrm{~d}^{-1}$ ), as previously suspected by Joshi et al. (2015). However, as revealed in the Lomb-Scargle spectral frequency distribution (Fig. B8, bottom), the range of interest (as shown in the inset) exhibits no clear signals above the noise level that would meet the false alarm probability (FAP) threshold criterion. In addition, the time-resolved radial velocity analysis did not reveal any short-term variability. Therefore, within the given photometric and spectroscopic detection limits, we conclude that HD 73045 is pulsationally stable. The RV values derived from our observations agree well with the previous measurements by Carquillat \& Prieur (2007). Indeed, the radial velocities, after being averaged over each series, are in good agreement with theoretical predictions based on the published stellar orbital parameters.

\subsection{HD 73574/KW 203 (Praesepe)}

HD 73574 is classified as A5V and A5III by Bidelman (1956) and Rebeirot (1966), respectively. Fossati et al. (2008) determined the values for $T_{\text {eff }}, \log g$ and $\left[\mathrm{F}_{\mathrm{e}} / \mathrm{H}\right]$ as $7662 \mathrm{~K}, 4.00(\mathrm{cgs})$ and 0.10 , respectively. This star was identified as a moderate rotator with $v \sin i$ equal to $120 \mathrm{~km} \mathrm{~s}^{-1}$ (McGee et al. 1967). The $K 2 \mathrm{C} 05$ data set exhibits two periods, namely 10.8 and 14.27 d (see Fig. B9). From the time-frequency as well as the ACF and CS analyses of the $K 2$ C05 data set, it is found that HD 73574 shows a periodicity of about $13.8 \mathrm{~d}$ (Fig. B20).

Using the spectroscopic observations, we derived values of $T_{\text {eff }}, \quad \log g,[\mathrm{M} / \mathrm{H}], \quad \xi, v \sin i$ and $\mathrm{RV}$ as $7700 \pm 160 \mathrm{~K}$, $4.12 \pm 0.21$ (cgs), $0.07 \pm 0.11,2.12 \pm 0.17 \mathrm{~km} \mathrm{~s}^{-1}, 99 \pm 5 \mathrm{~km} \mathrm{~s}^{-1}$, and $29.2 \pm 2.8 \mathrm{~km} \mathrm{~s}^{-1}$, respectively.

\subsection{HD 73618/KW 224 (Praesepe)}

HD 73618 is classified as A0 spectral type (Cannon \& Pickering 1993). It is also referred to as an Am star (Bidelman 1956; Bertaud 1960; Rebeirot 1966). The $v \sin i$ value for this star has been reported as $51 \mathrm{~km} \mathrm{~s}^{-1}$ (Abt \& Willmarth 1999) and $44 \mathrm{~km} \mathrm{~s}^{-1}$ (Debernardi et al. 2000).

Mason et al. (1993) identified this star as the primary component of a SB1 binary system in which the flux of the secondary star does not significantly influence the combined total flux spectrum. There are various studies about the atmospheric parameters. Based on those determinations, $T_{\mathrm{eff}}, \log g$, and $\left[\mathrm{F}_{\mathrm{e}} / \mathrm{H}\right]$ are given as $8060 \mathrm{~K}$, 3.87 (cgs), and 0.46 by Hui-Bon-Hoa, Burkhart \& Alecian (1997); $8100 \mathrm{~K}, 4.00$ (cgs), and 0.50 by Burkhart \& Coupry (1998); and $8170 \mathrm{~K}, 4.00$ (cgs), and 0.46 by Fossati et al. (2007). Fossati et al. (2007) did not find any detectable magnetic field.

The K2 C05 data set (Fig. C11) shows a dominant peak at $3.78 \mathrm{~d}$. In the $\mathrm{C} 16$ data set (Fig. C12), the three peaks are given as 3.83, 4.87, and $2.49 \mathrm{~d}$, whereas in the $\mathrm{C} 18$ data set (Fig. C13), they are given as 3.76, 4.81, and $2.37 \mathrm{~d}$. These main features are accompanied by lower-amplitude, seemingly unrelated, components, producing a rather complex time-series. The time-frequency, ACF and CS analyses suggest a periodic variability of about $4 \mathrm{~d}$, with its origin still undetermined.

An attempt was made to analyse the $\mathrm{C} 16$ and $\mathrm{C} 18$ time-series together, in a 208-d data set that includes a non-centred gap extending across 36 percent of the temporal baseline. While issues arise when applying Fourier methods to gapped data (Munteanu et al. 2016), the Lomb-Scargle algorithm did produce a periodogram with a frequency resolution of $0.05 \mathrm{~d}^{-1}$. Applying oversampling to the Lomb-Scargle process introduced sidelobes that completely corrupted the periodogram.

Based on our spectroscopic analysis, the derived parameters are given as $T_{\text {eff }}=7960 \pm 180 \mathrm{~K}, \log g=3.76 \pm 0.19(\mathrm{cgs}),[\mathrm{M} / \mathrm{H}]=$ 
$0.34 \pm 0.11, \xi=2.77 \pm 0.16 \mathrm{~km} \mathrm{~s}^{-1}, v \sin i=56 \pm 3 \mathrm{~km} \mathrm{~s}^{-1}$, and $\mathrm{RV}=39.5 \pm 0.5 \mathrm{~km} \mathrm{~s}^{-1}$. Based on age and metallicity, Ahumada \& Lapasset (2007) classified HD 73618 as a blue straggler; hence, it can be considered an ideal object for testing current models of collisionally formed blue stragglers. The wide and detailed knowledge available on this star and its environment should allow us to test the reliability of current models and provide important constraints for future model development.

\subsection{HD 73619/KW 229 (Praesepe)}

HD 73619 is a member of the Praesepe open cluster (Sanford 1931). Bidelman (1956) identified this star as a classical Am star, with a spectral type given as A4 and F0 III based on Ca II K and metallic lines, respectively. Abt \& Willmarth (1999) and Debernardi et al. (2000) identified the projected stellar rotational velocity as 20 and $11.2 \mathrm{~km} \mathrm{~s}^{-1}$, respectively.

The light curve of HD 73619 shown in the upper left-hand panel of Fig. C14 is similar to that of a heartbeat star (Thompson et al. 2012; Fuller 2017; Hambleton et al. 2018; Wheeler \& Kipping 2019; van Kooten, Kenworthy \& Doelman 2020). Analysis of the frequency spectrum provided orbital periodicities of 12.97 and $12.91 \mathrm{~d}$ from the $\mathrm{C} 05$ and $\mathrm{C} 18$ data, respectively. These values were confirmed by using time-domain autocorrelation and box-fitting (Kovács, Zucker \& Mazeh 2002) methods. Although HD 73619 was observed during $\mathrm{C} 16$ of the $K 2$ mission, its image was blended with HD 73598; thus, these C16 observations have been excluded from this study.

A thorough search for any pulsational variability induced by tidal interaction requires an accurate model to be constructed and subtracted from the light curve, and the residuals to be analysed. While conservative sinusoidal models were produced from a limited number of components (Table 3), many more components would be required to reconstruct the light curve accurately. None of these components relates to pulsational signals. Construction of a detailed model was also attempted using the PHOEBE package (Prša \& Zwitter 2005), but the procedure failed to converge. However, two other methods were performed to probe the existence of pulsational phenomena.

One method involved separating the heartbeat light curve into separate intra-period segments. Each segment was fitted with an eighth-order polynomial, which was subsequently subtracted from the data, resulting in respective sets of residuals (Fig. 12, top lefthand panel). These residual data sets were analysed with the LombScargle algorithm to produce a frequency spectrum (Fig. 12, top right-hand panel). While an instrumental artefact appeared in the third and fourth segments of the C05 data, evidence of pulsation was not observed, and nor was any such evidence seen in the C18 data (Fig. C29). The other method involved using the K2SC algorithm previously applied to clean SAP light curves. This process also produced a de-trended residual data set with the dominant heartbeat signal removed (Fig. 12, middle). Again, the data sets were analysed using the Lomb-Scargle algorithm with no evidence of pulsation signals found either in the C05 (Fig. 12, bottom) or in the C18 data (Fig. C29, middle and bottom). In conclusion, within the limits of our analysis, HD 73619 shows no evidence of pulsational variability.

Based on the parameters given in Table 5, we ratify that the two components of the heartbeat system have similar characteristics. From Fig. 4, our revised radial velocities support the orbital solution determined by Debernardi et al. (2000). Moreover, Debernardi et al. (2000) reported an orbital period of $12.91124 \pm 0.00004 \mathrm{~d}$. This implies that the dominant 12.91-d signal detected in both $K 2$ data sets is orbital in nature. The spectropolarimetric analysis indicates that, within the observational uncertainty of $200 \mathrm{G}$, magnetic fields in HD 73619 are either weak or absent.

\subsection{HD 76310}

Cowley \& Cowley (1965b) classified HD 76310 as an Am star. The projected rotational velocity $v \sin i$ as a function of the predicted equatorial rotational velocity $v_{\text {rot }}$ for the target stars is shown in Fig. 8. The blue, green, and black dashed lines correspond to the inclination angles $i=90^{\circ}, 60^{\circ}$, and $30^{\circ}$, respectively. Note that HD 73619 is not included in this diagram because its photometric signal is orbital in nature.

All three $K 2$ data sets tend to be dominated by two components at $\sim 4.7$ and $\sim 5.4 \mathrm{~d}$, with variation around those values across the campaigns. These close frequencies result in well-pronounced beating effects in $\mathrm{C} 05$ (Fig. B16) and C16 (Fig. B17), but less interaction between the components during C18 (Fig. B18), for which the shape of the time-series varies across the periods. The level of these interactions is defined by the ratio of their amplitudes. Also note that overtones of the $\sim 4$.8-d feature seem to exist, although they do not always clear the FAP threshold and, in some cases, are obscured by the background noise. Finally, it is noteworthy that the modelling of the $\mathrm{C} 05$ time-series failed to produce a good fit to the data when the single component relating to the dominant 4.622-d peak was included, and two other components at around $\pm 0.3 \mathrm{~d}$ either side of the peak were required to better fit the data. From the time-series analysis of the $K 2$ data, the surface map, the wavelet, the $\mathrm{ACF}$, and the composite spectrum, a dominant period close to $4.7 \mathrm{~d}$ was obtained. Given that the photometric amplitude of HD 73610 changes significantly within only a few dozen variability cycles, we cannot rule out the possibility of having unresolved multiperiodic pulsational signals, for instance of the $\gamma$ Doradus type. An attempt to analyse the combined time-series of C16 and C18, corresponding to that for HD 73618, was performed with a similar outcome.

On investigating the $v \sin i$ versus $v_{\text {rot }}$ diagram in Fig. 8, HD 76310 was found to be positioned below the line of $\sin i=1$. Based on the surface maps, wavelet, and ACF analyses, we thus categorize this star as a rotational variable. The LSD profiles shown in Fig. 13 indicate a complex structure. The RV values obtained at different epochs (2018 December and 2020 April) are the same within the observational uncertainty (i.e. $20.13 \pm 0.40$ and $20.22 \pm 0.83 \mathrm{~km} \mathrm{~s}^{-1}$, respectively). The similarity between the shapes of the LSD profile of HD 76310 and the cross correlation function (CCF) profile of KIC 11572666 (Lampens et al. 2018) implies that the system is an SB2 system. We fitted the MRES spectrum of HD 76310 with a composite model using the $2 \mathrm{D}$ version of GIRFIT in spectral regions around $\mathrm{H} \gamma(430-440 \mathrm{~nm}), \mathrm{H} \beta(470-500 \mathrm{~nm}), \mathrm{H} \alpha(630-680 \mathrm{~nm})$, and the Mg I triplet (510-520 nm). We treated the component $T_{\text {eff }}, v \sin i$, $\mathrm{RV}$ and the luminosity ratio as free parameters while keeping $\log g$ fixed at $4.0(\mathrm{cgs})$.

The composite spectrum was fitted well (e.g. see the $\mathrm{H} \beta$ line in Fig. 14); thus, we adopted the mean values of the stellar parameters from these spectral regions. The obtained values are: effective temperature of $T_{\text {eff } 1}=7030 \pm 170 \mathrm{~K}$ and $T_{\text {eff } 2}=6470 \pm 220 \mathrm{~K}, v \sin i$ of $97.1 \pm 0.4 \mathrm{~km} \mathrm{~s}^{-1}$ and $9.3 \pm 0.7 \mathrm{~km} \mathrm{~s}^{-1}$, RV of $27.4 \pm 1.8 \mathrm{~km} \mathrm{~s}^{-1}$ and $19.8 \pm 2.8 \mathrm{~km} \mathrm{~s}^{-1}$, respectively, with a light ratio $l_{1}$ of $0.887 \pm 0.023$ (see Table 5).

Based on the rotational velocities and periods of HD 76310, the source of the stellar photometric variability, as indicated, could be 

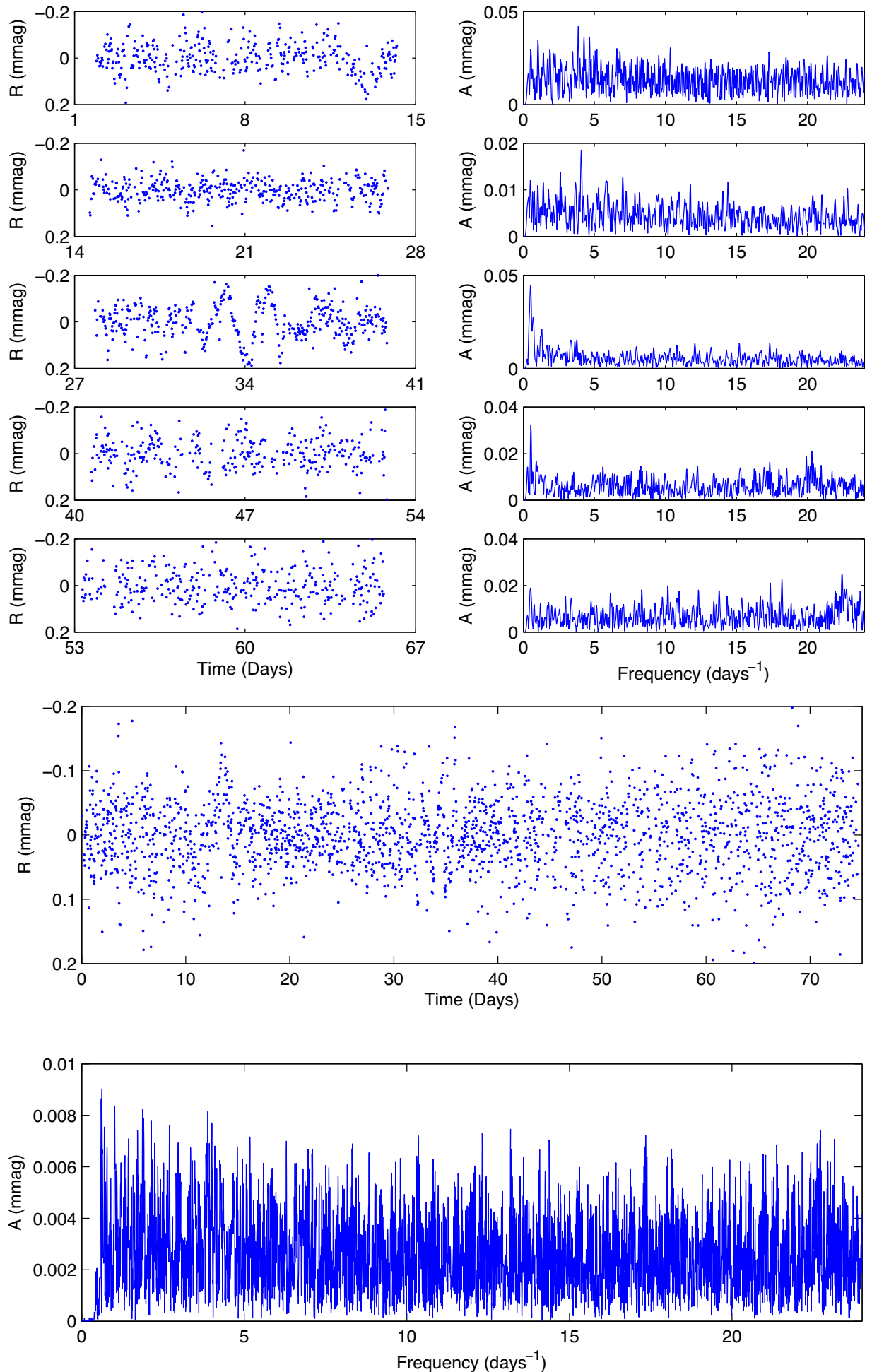

Figure 12. The top five rows illustrate the residual light curve (left-hand panels) and amplitude spectrum (right-hand panels) of HD 73619 after removing the heartbeat signal from five segments of the $\mathrm{C} 05$ time-series. The $\sim 0.5-\mathrm{d}^{-1}$ spectral peaks in segments 3 and 4 are related to an instrumental artefact. The middle panel shows the complete time-series after de-trending by the K2SC algorithm, with its respective amplitude spectrum in the bottom panel. Neither analysis indicates any evidence for the existence of pulsation phenomena.

the sharp-lined and cooler component, thus favouring the stellar binarity hypothesis. It is also noteworthy that HD 76310 is included in the Washington Double Star Catalogue (Mason et al. 2001) as a system consisting of two equally bright stars separated by 0.1 arcsec. Nevertheless, there is still the possibility that HD 76310 is a single star surrounded by a cloud shell (Slettebak 1986; Fekel, Warner \& 


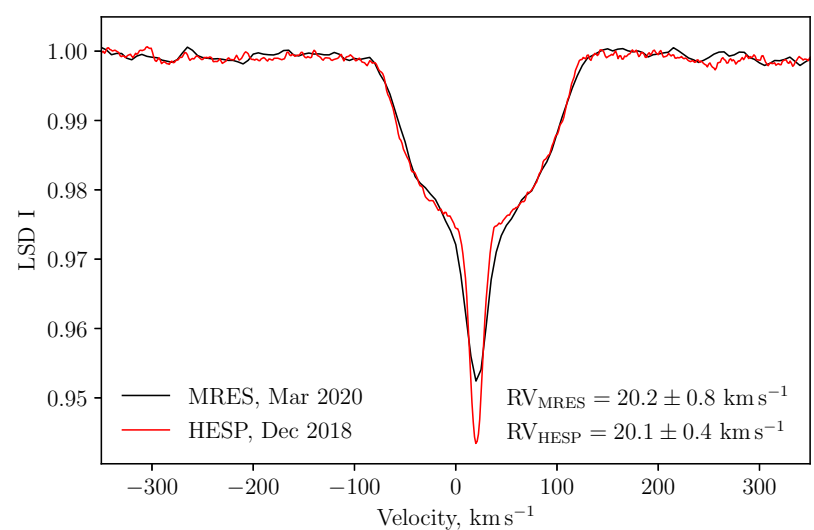

Figure 13. The LSD profiles for HD 76310 based on HESP (red) and MRES (black) data.
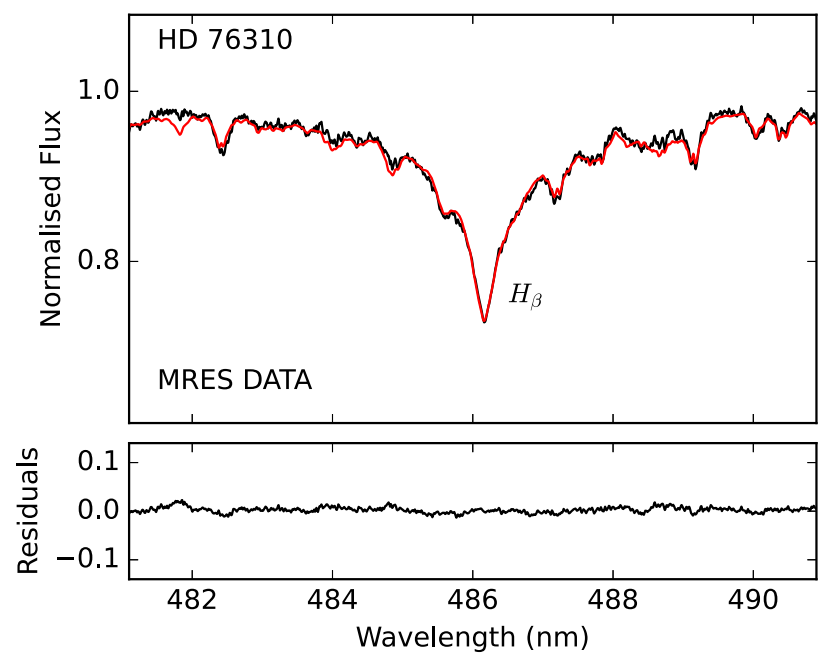

Figure 14. The $\mathrm{H} \beta$ MRES spectrum for HD 76310 (black) and the composite model spectrum (red) obtained using the 2D version of GIRFIT.

Kaye 2003). Future observations will be crucial for exploring the variability and possible binary nature of this object (Welsh \& Montgomery 2013).

\section{CONCLUSIONS AND FUTURE PROSPECTS}

Based on the time-series photometry obtained from the ground, the $K 2$ mission, and contemporaneous high-resolution spectroscopy and spectropolarimetry, we arrived at the following conclusions.

(i) All targets in the study sample, previously part of the NainitalCape survey and classified as constant stars, are now (potentially) identified as variables. Our analysis suggests that HD 73045 and HD 76310 are rotational variables. The LSD profile of HD 76310 indicates that this star is part of a binary system or is surrounded by a cloud shell. The light variation recorded for HD 73619 is consistent with orbital signals and likely to be the first CP Am star in a heartbeat system without tidally induced pulsations. As we could not ascertain the origin of the variability of HD 73574 and HD 73618, further timedomain studies are needed to ascertain the nature of these objects.

(ii) Within the detection limits of the $K 2$ photometry and radial velocity measurements, we conclude that HD 73045 is pulsationally stable, contrary to the previous prediction by Joshi et al. (2015).
Similarly, the analysis of the $K 2$ data revealed the absence of the periodicity of 1.25 d previously reported by Paunzen et al. (2013); the previous results are confirmed to be erroneous.

(iii) High-resolution spectroscopy measurements obtained with two spectrographs at different epochs revealed that the HD 73619 system comprises two stars with similar properties. The individual radial velocities of HD 73619 at the different epochs are in good agreement with the orbital solution by Debernardi et al. (2000). The spectropolarimetric data analysis indicates that HD 73619 may possess a weak magnetic field, and hence additional high-resolution spectropolarimetric observations are essential for confirmation.

(iv) The atmospheric parameters of the target stars have been derived using high-resolution spectroscopy. Considering observational uncertainties, it is found that all the studied stars are evolved away from the ZAMS and located within the $\delta$ Scuti instability strip.

The potential presence of a weak magnetic field in HD 73619, and the surface spots seen on HD 73045 and HD 76310 constitute further evidence that magnetic fields cannot be disregarded when exploring the origin of the peculiar chemical abundances in Am stars. The magnetic fields leading to the formation of the surface spots might not be stable, suggesting that they may be dominated by the dynamo effect. It is unclear if (or how) magnetic fields would affect the diffusion model for the formation of the peculiar chemical abundances in these stars, or whether alternative formation mechanism(s) would be needed to explain these phenomena.

The origin of weak magnetic fields in metallic-line (Am) stars and even in normal $\delta$ Sct stars (hotter than the Sun), thought to be fossil or related to a dynamo effect, is still largely an unsolved problem, although copious valuable contributions for A-type stars have previously been made (e.g. MacDonald \& Mullan 2004). A detailed study of the various underlying processes, particularly the diverse theoretical aspects, is required. Future studies are relevant to the bigger picture of stellar and galactic evolution, in consideration of the significance of massive stars, notably for the production and proliferation of heavy elements as well as the overall galactic chemistry (e.g. Matteucci 2021, and references therein).

Our overall long-term goal in this work is to continue the study of stellar structure and atmospheres of CP stars as well as of magnetic fields, inhomogeneities (such as spots), and tidal interaction. In the framework of our ongoing research, our future plan is to extend the detailed analysis of heretofore unstudied CP stars with TESS data in combination with future high-resolution spectroscopic and spectropolarimetric observations.

\section{ACKNOWLEDGEMENTS}

The work presented here is supported by the Belgo-Indian Network for Astronomy and Astrophysics (BINA), sanctioned by the Department of Science and Technology (DST, Government of India; DST/INT/Belg/P-09/2017) and the Belgian Federal Science Policy Office (BELSPO, Government of Belgium; BL/33/IN12). OT and EJ thank the International Science Programme (ISP) of Uppsala University and African Astronomical Society (AfAS) for financial support. DLH acknowledges financial support from the Science and Technology Facilities Council (STFC) via grant ST/M000877/1. AG, DM, and SJ are grateful for the support received from the IndoThailand Programme of co-operation in Science and Technology through the Indo-Thai joint project DST/INT/Thai/P-16/2019. FKA and SC thank the Polish National Center for Science (NCN) for grant 2015/18/A/ST9/00578. RAG acknowledges support from the 
CNES PLATO grant. SM acknowledges support from the Spanish Ministry with the Ramony Cajal fellowship number RYC-201517697. ARGS acknowledges support from NASA under grant no. NNX17AF27G and from STFC consolidated grant ST/T000252/1. IS thanks the Government of the Russian Federation and the Ministry of Higher Education and Science of the Russian Federation, grant no. 075-15-2020-780 (N13.1902.21.0039). This paper includes data collected by the $K 2$ mission available at Mikulski Archive for Space Telescopes (MAST), SIMBAD and NASA's ADS. The authors thank the anonymous reviewer for the insightful comments and suggestions which led to improvements in the manuscript.

\section{DATA AVAILABILITY}

The data underlying this article will be shared on reasonable request to the corresponding author.

\section{REFERENCES}

Abt H. A., Willmarth D. W., 1999, ApJ, 521, 682

Ahumada J. A., Lapasset E., 2007, A\&A, 463, 789

Aigrain S., Hodgkin S. T., Irwin M. J., Lewis J. R., Roberts S. J., 2015, MNRAS, 447, 2880

Aigrain S., Parviainen H., Pope B. J. S., 2016, MNRAS, 459, 2408

Antoci V. et al., 2019, MNRAS, 490, 4040

Ashoka B. N. et al., 2000, Bull. Astron. Soc. India, 28, 251

Asplund M., Grevesse N., Sauval A. J., Scott P., 2009, ARA\&A, 47, 481

Bagnulo S., Szeifert T., Wade G. A., Landstreet J. D., Mathys G., 2002, A\&A, 389,191

Balona L. A., 2011, MNRAS, 415, 1691

Balona L. A., 2013, MNRAS, 431, 2240

Balona L. A., 2017, MNRAS, 467, 1830

Balona L. A., 2018, MNRAS, 479, 183

Balona L. A., Catanzaro G., Abedigamba O. P., Ripepi V., Smalley B., 2015, MNRAS, 448, 1378

Beck P. G. et al., 2014, A\&A, 564, A36

Bertaud C., 1960, J. Obser., 43, 129

Bidelman W. P., 1956, PASP, 68, 318

Blanco-Cuaresma S., 2019, MNRAS, 486, 2075

Blanco-Cuaresma S., Soubiran C., Jofré P., Heiter U., 2014, A\&A, 569, 111

Blazère A. et al., 2015, in Nagendra K. N., Bagnulo S., Centeno R., Jesús Martínez González M., eds, Proc. IAU Symp. 305, Polarimetry: From the Sun to Stars and Stellar Environments. Cambridge Univ. Press, Cambridge, p.67

Blazère A., Neiner C., Petit P., 2016, MNRAS, 459, L81

Böhm T. et al., 2015, A\&A, 577, A64

Bowman D. M., Buysschaert B., Neiner C., Pápics P. I., Oksala M. E., Aerts C., 2018, A\&A, 616, A77

Burdanov A. Y., Krushinsky V. V., Popov A. A., 2014, Astrophys. Bull., 69, 368

Burkart J., Quataert E., Arras P., Weinberg N. N., 2012, MNRAS, 421, 983

Burkhart C., Coupry M. F., 1998, A\&A, 338, 1073

Cannon A. J., Pickering E. C., 1993, VizieR Online Data Catalog, p. III/135A

Carquillat J.-M., Prieur J.-L., 2007, MNRAS, 380, 1064

Castelli F., Kurucz R. L., 2003, in Piskunov N., Weiss W. W., Gray D. F., eds, IAU Symp. Vol. 210, Modelling of Stellar Atmospheres, Astron. Soc. Pac., San Francisc. p. A20

Ceillier T. et al., 2016, MNRAS, 456, 119

Ceillier T. et al., 2017, A\&A, 605, A111

Chanumolu A., Jones D., Thirupathi S., 2015, Exp. Astron., 39, 423

Chountonov G. A., 2004, in Arkhyz N., Glagolevskij Y. V., Kudryavtsev I. I., Romanyuk I. I., eds, Magnetic stars, Proceedings of the International Conference, held in the Special Astrophysical Observatory of the Russian AS, August 27-31, 2003, p. 286

Cowley A. P., Cowley C. R., 1965a, PASP, 77, 184
Cowley A. P., Cowley C. R., 1965b, PASP, 77, 184

Crawford D. L., Mandwewala N., 1976, PASP, 88, 917

Cutri R. M. et al., 2003, VizieR Online Data Catalog, 2MASS All-Sky Catalogof Point Sources, p. II/246

Davis L. E., 1994, in Crabtre D. R., Hanisch R. J., Barnes J., eds, Astronomical Data Analysis Softwareand Systems II, ASP Conf. Ser., 61. Astronomical Society of the Pacific, San Francisco, p. 75

Debernardi Y., Mermilliod J.-C., Carquillat J.-M., Ginestet N., 2000, A\&A, 354,881

Driesman A., Hynes S., Cancro G., 2008, Space Sci. Rev., 136, 17

Eyles C. J. et al., 2009, Sol. Phys., 254, 387

Fekel F. C., Warner P. B., Kaye A. B., 2003, AJ, 125, 2196

Fossati L., Bagnulo S., Landstreet J., Wade G., Kochukhov O., Monier R., Weiss W., Gebran M., 2008, A\&A, 483, 891

Fossati L., Bagnulo S., Monier R., Khan S. A., Kochukhov O., Landstreet J., Wade G., Weiss W., 2007, A\&A, 476, 911

Frémat Y., Neiner C., Hubert A. M., Floquet M., Zorec J., Janot-Pacheco E., Renan de Medeiros J., 2006, A\&A, 451, 1053

Fuller J., 2017, MNRAS, 472, 1538

Fuller J., Lai D., 2012, MNRAS, 420, 3126

Gaia Collaboration et al., 2018, A\&A, 616, A1

García R. A. et al., 2014, A\&A, 572, A34

García R. A., Mathur S., Salabert D., Ballot J., Régulo C., Metcalfe T. S., Baglin A., 2010, Science, 329, 1032

Giles H. A. C., Collier Cameron A., Haywood R. D., 2017, MNRAS, 472, 1618

Girardi L., Grebel E. K., Odenkirchen M., Chiosi C., 2004, A\&A, 422, 205

Goupillaud P., Grossmann A., Morlet J., 1984, Geoexploration, 23, 85

Green G. M., 2018, Journal of Open Source Software, 3, 695

Green G. M., Schlafly E. F., Zucker C., Speagle J. S., Finkbeiner D. P., 2019, ApJ, 887, 93

Guo Z., Gies D. R., Fuller J., 2017, ApJ, 834, 59

Guo Z., Shporer A., Hambleton K., Isaacson H., 2020, ApJ, 888, 95

Guzik J. A., 2021, Frontiers Astron. Space Sci., 8, 55

Hambleton K. et al., 2016, MNRAS, 463, 1199

Hambleton K. et al., 2018, MNRAS, 473, 5165

Hambleton K. M. et al., 2013, MNRAS, 434, 925

Handler G. et al., 2002, MNRAS, 333, 262

Heiter U. et al., 2015, Physica Scripta, 90, 4010

Hill G. M., Bohlender D. A., Landstreet J. D., Wade G. A., Manset N., Bastien P., 1998, MNRAS, 297, 236

Høg E. et al., 2000, A\&A, 355, L27

Holschneider M., Kronland-Martinet R., Morlet J., Tchamitchian P., 1989, in Combes J.-M., Grossmann A., Tchamitchian P., eds, Wavelets. TimeFrequency Methods and Phase Space. IPTI, Springer-Verlag Berlin Heidelberg, p. 286

Howell S. B. et al., 2014, PASP, 126, 398

Hui-Bon-Hoa A., Burkhart C., Alecian G., 1997, A\&A, 323, 901

Joshi S. et al., 2003, MNRAS, 344, 431

Joshi S. et al., 2012, MNRAS, 424, 2002

Joshi S. et al., 2016, A\&A, 590, A116

Joshi S., Joshi G. C., Joshi Y. C., Aggrawal R., 2015, in Meynet G., Georgy C., Groh J., Stee P., eds, New windows on massive stars: asteroseismology, interferometry, and spectropolarimetry, Proceedings of the International Astronomical Union, IAU Symposium, 307, p. 218

Joshi S., Mary D. L., Chakradhari N. K., Tiwari S. K., Billaud C., 2009, A\&A, 507, 1763

Joshi S., Mary D. L., Martinez P., Kurtz D. W., Girish V., Seetha S., Sagar R., Ashoka B. N., 2006, A\&A, 455, 303

Joshi S., Ryabchikova T., Kochukhov O., Sachkov M., Tiwari S. K., Chakradhari N. K., Piskunov N., 2010, MNRAS, 401, 1299

Joshi S., Semenko E., Moiseeva A., Sharma K., Joshi Y. C., Sachkov M., Singh H. P., Yerra B. K., 2017, MNRAS, 467, 633

Kirk B. et al., 2016, AJ, 151, 68

Kochukhov O., Bagnulo S., Wade G. A., Sangalli L., Piskunov N., Landstreet J. D., Petit P., Sigut T. A. A., 2004, A\&A, 414, 613

Kochukhov O., Makaganiuk V., Piskunov N., 2010, A\&A, 524, A5 
Kołaczek-Szymański P. A., Pigulski A., Michalska G., Moździerski D. Różański T., 2021, A\&A, 447, 12

Kovács G., Zucker S., Mazeh T., 2002, A\&A, 391, 369

Kraus A. L., Hillenbrand L. A., 2007, AJ, 134, 2340

Kudryavtsev D. O., Romanyuk I. I., Elkin V. G., Paunzen E., 2006, MNRAS, 372, 1804

Kumar P., Ao C. O., Quataert E. J., 1995, ApJ, 449, 294

Kupka F., 2003, eds, Balona L. A., Henrichs H. F., Medupe R., in Magnetic Fields in O, B and A Stars: Origin and Connection to Pulsation, Rotation and Mass Loss, Vol. 305, ASP Conf. Ser., Astronomical Society of the Pacific, San Francisco, p. 190

Lampens P. et al., 2018, A\&A, 610, A17

Lipunov V. et al., 2010, Adv. Astron., 2010, 349171

MacDonald J., Mullan D. J., 2004, MNRAS, 348, 702

Maceroni C. et al., 2009, A\&A, 508, 1375

Martinez P. et al., 2001, A\&A, 371, 1048

Martínez-Arnáiz R., Maldonado J., Montes D., Eiroa C., Montesinos B., 2010, A\&A, 520, A79

Mason B. D., Hartkopf W. I., McAlister H. A., Sowell J. R., 1993, AJ, 106, 637

Mason B. D., Wycoff G. L., Hartkopf W. I., Douglass G. G., Worley C. E., 2001, AJ, 122, 3466

Mathur S. et al., 2010, A\&A, 511, A46

Mathur S. et al., 2014, A\&A, 562, A124

Matteucci F., 2021, A\&A Rev., 29, 5

McGee J. D., Khogali A., Baum W. A., Kraft R. P., 1967, MNRAS, 137, 303

McQuillan A., Aigrain S., Mazeh T., 2013, MNRAS, 432, 1203

McQuillan A., Mazeh T., Aigrain S., 2014, ApJS, 211, 24

Mermilliod J.-C., Mermilliod M., Hauck B., 1997, A\&AS, 124, 349

Michaud G., 1970, ApJ, 160, 641

Michaud G., Charland Y., Megessier C., 1981, A\&A, 103, 244

Munteanu C., Negrea C., Echim M., Mursula K., 2016, Ann. Geophys., 34, 437

Murphy S. J., Hey D., Van Reeth T., Bedding T. R., 2019, MNRAS, 485, 2380

Neiner C., Lampens P., 2015, MNRAS, 454, L86

Neiner C., Wade G. A., Sikora J., 2017, MNRAS, 468, L46

O’Leary R. M., Burkart J., 2014, MNRAS, 440, 3036

Pamyatnykh A. A., 2000, in Breger M., Montgomery M., eds, Proceedings of the 6th Vienna Workshop in Astrophysics, held in Vienna, Austria, 4-7 August 1999, ASP Conference Series, 210. ASP, San Francisco, p. 215

Panchuk V. E., Chuntonov G. A., Naidenov I. D., 2014, Astrophys. Bull., 69, 339

Panchuk V. E., Klochkova V. G., Yushkin M. V., 2017, Astron. Rep., 61, 820

Paunzen E., Wraight K. T., Fossati L., Netopil M., White G. J., Bewsher D., 2013, MNRAS, 429, 119

Petit P. et al., 2011, A\&A, 532, L13

Preston G. W., 1974, ARA\&A, 12, 257

Prša A., Zwitter T., 2005, ApJ, 628, 426

Raskin G. et al., 2011, A\&A, 526, A69

Rebeirot E., 1966, Publ. Obs. Haute-Provence, 8, 24

Sagar R. et al., 2011, Current Sci., 101, 1020

Sanford R. F., 1931, ApJ, 74, 201

Santos A. R. G., Cunha M. S., Avelino P. P., García R. A., Mathur S., 2017, A\&A, 599, A1

Santos A. R. G., García R. A., Mathur S., Bugnet L., van Saders J. L., Metcalfe T. S., Simonian G. V. A., Pinsonneault M. H., 2019, ApJS, 244, 21

Santos A. R. G., Mathur S., García R. A., Cunha M. S., Avelino P. P., 2021, MNRAS, 508, 268

Savanov I. S., 2018, Astron. Rep., 62, 814

Savanov I. S., Strassmeier K. G., 2008, Astron. Nachrichten, 329, 364

Sinvhal S. D., Kandpal C. D., Mahra H. S., Joshi S. C., Srivastava J. B., 1972, in Optical Astronomy with Moderate Size Telescopes, Symposium held at Centre of Advanced Study in Astronomy, 64. Osmania Univ., Hyderabad, p. 20

Slettebak A., 1986, PASP, 98, 867

Smalley B. et al., 2017, MNRAS, 465, 2662
Smith J. C. et al., 2012, PASP, 124, 1000

Sneden C., Bean J., Ivans I., Lucatello S., Sobeck J., 2012, MOOG: LTE line analysis and spectrum synthesis, Astrophysics Source Code Library, ascl.net/1202.009

Sriram S. et al., 2018, Proceedings of the SPIE, Volume 10702, p. 107026K Stumpe M. C. et al., 2012, PASP, 124, 985

Thompson S. E. et al., 2012, ApJ, 753, 86

Torrence C., Compo G. P., 1998, Bull. Am. Meteorological Soc., 79, 61

Trust O., Jurua E., De Cat P., Joshi S., 2020, MNRAS, 492, 3143

Trust O., Jurua E., De Cat P., Joshi S., Lampens P., 2021, MNRAS, 504, 5528

van Kooten M. A. M., Kenworthy M., Doelman N., 2020, MNRAS, 499, 2817

Welsh B. Y., Montgomery S., 2013, PASP, 125, 759

Welsh W. F. et al., 2011, ApJS, 197, 4

Wheeler A., Kipping D., 2019, MNRAS, 485, 5498

White T. R. et al., 2017, MNRAS, 471, 2882

\section{SUPPORTING INFORMATION}

Supplementary data are available at MNRAS online.

\section{Joshi_Study_CP_Stars_Supplementary.pdf}

Please note: Oxford University Press is not responsible for the content or functionality of any supporting materials supplied by the authors. Any queries (other than missing material) should be directed to the corresponding author for the article.

${ }^{1}$ Aryabhatta Research Institute of Observational Sciences, Manora Peak, Nainital-263002, India

${ }^{2}$ Department of Physics, Mbarara University of Science and Technology, P.O. Box 1410, Mbarara, Uganda

${ }^{3}$ National Astronomical Research Institute of Thailand, Chiangmai 50180, Thailand

${ }^{4}$ Special Astrophysical Observatory, Russian Academy of Sciences, Nizhnii Arkhyz 369167, Russia

${ }^{5}$ Affiliate Faculty, Physics and Astronomy Department, George Mason University, Fairfax, VA 22030, USA

${ }^{6}$ Royal Observatory of Belgium, Ringlaan 3, B-1180 Brussels, Belgium

${ }^{7}$ Jeremiah Horrocks Institute, University of Central Lancashire, Preston PRI $2 H E, U K$

${ }^{8}$ CEA, Université Paris-Saclay, F-91191 Gif-sur-Yvette, France

${ }^{9}$ AIM, CEA, CNRS, Université Paris-Saclay, Université Paris Diderot, Sorbonne Paris Cité, F-91191 Gif-sur-Yvette, France

${ }^{10}$ Universidad de La Laguna (ULL), Departamento de Astrofísica, E-38206 La Laguna, Tenerife, Spain

${ }^{11}$ Instituto de Astrofísica de Canarias (IAC), E-38205 La Laguna, Tenerife, Spain

${ }^{12}$ Department of Physics, University of Warwick, Coventry CV4 7AL, UK

${ }^{13}$ Space Science Institute, 4765 Walnut Street, Suite B, Boulder, CO 80301, USA

${ }^{14}$ Indian Institute of Astrophysics, Koramangala, Bangalore-560034, India

${ }^{15}$ Department of Physics, University of Texas at Arlington, Arlington, TX 76019, USA

${ }^{16}$ Department of Physics and Astronomy, National Institute of Technology, Rourkela-769008, Odisha, India

${ }^{17}$ Copernicus Astronomical Center, Bartycka 18, PL-00-716 Warsaw, Poland

${ }^{18}$ Çanakkale Onsekiz Mart University, Faculty of Sciences and Arts, Physics Department, 17100 Canakkale, Turkey

${ }^{19}$ Centre for Space Research, North-West University, Potchefstroom, 2531, South Africa

${ }^{20}$ Stellar Astrophysics Centre, Department of Physics and Astronomy, Aarhus University, Ny Munkegade 120, 8000, Denmark

${ }^{21}$ Institute of Astronomy, Russian Academy of Sciences, Moscow-119017, Russia 
${ }^{22}$ Institut d'Astronomie et d'Astrophysique, Université Libre de Bruxelles, Boulevard du Triomphe, 1050 Brussels, Belgium

${ }^{23}$ Kourovka Astronomical Observatory, Ural Federal University, Yekaterinburg, 620000, Russia

${ }^{24}$ M. V. Lomonosov Moscow State University, P. K. Shternberg State Astronomical Institute, Moscow, 119234, Russia

${ }^{25}$ School of Studies in Physics and Astrophysics, Pt. R. S. University, Raipur492010, India
${ }^{26}$ Institute of Informatics and Communication, University of Delhi South Campus, New Delhi, 110021, India

${ }^{27}$ Department of Physics, Kyambogo University, P.O. Box 1, Kyambogo, Kampala, Uganda

${ }^{28}$ Indian Institute of Science (IISc), Bengaluru, 560012, India

This paper has been typeset from a $\mathrm{T}_{\mathrm{E}} \mathrm{X} / \mathrm{LT} \mathrm{E} \mathrm{X}$ file prepared by the author. 